THESIS FOR THE DEGREE OF DOCTOR OF PHILOSOPHY

\title{
The death throes of massive stars
}

\author{
SOFIA WALLSTRÖM
}

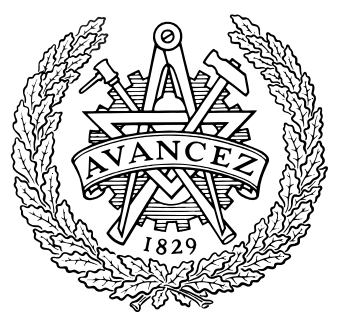

CHALMERS

Department of Earth and Space Sciences CHALMERS UNIVERSITY OF TECHNOLOGY

Göteborg, Sweden 2016 
The death throes of massive stars

SOFIA WALLSTRÖM

ISBN 978-91-7597-371-5

(C) Sofia Wallström, 2016

Doktorsavhandlingar vid Chalmers tekniska högskola

Ny serie nr 4052

ISSN: 0346-718X

Radio Astronomy \& Astrophysics Group

Department of Earth and Space Sciences

Chalmers University of Technology

SE-412 96 Göteborg, Sweden

Phone: +46 (0)31-772 1000

\section{Contact information:}

Sofia Wallström

Onsala Space Observatory

Chalmers University of Technology

SE-439 92 Onsala, Sweden

Phone: +46 (0)31-772 5544

Fax: $\quad+46(0) 31-7725590$

Email: sofia.wallstrom@chalmers.se

\section{Cover image:}

Spectra over the Herschel PACS footprint, showing CO J=23-22 in blue and [OIII] $88 \mu \mathrm{m}$ in red, overlaid on a Spitzer/IRAC image of the CO vibrational emission in Cas A. Image credit: Wallström et al., 2013

Printed by Chalmers Reproservice Chalmers University of Technology

Göteborg, Sweden 2016 


\title{
The death throes of massive stars
}

\author{
SOFIA WALLSTRÖM \\ Department of Earth and Space Sciences \\ Chalmers University of Technology
}

\begin{abstract}
Massive evolved stars affect their local surroundings as they go through phases of intense mass-loss and eventually explode as supernovae, adding kinetic energy and freshly synthesised material to the interstellar medium. The circumstellar material ejected by the star affects the shape and evolution of the future supernova remnant, and how the material is incorporated into the interstellar medium. Over time, these processes affect the chemical evolution of the interstellar medium on a galactic scale. This thesis probes these topics at different stages.

First, observations of CO lines in the supernova remnant Cassiopeia A have been used to study the effect of the reverse shock on supernova ejecta (Paper I). We find a large column density of warm $\mathrm{CO}$, which has most likely re-formed after the passage of the reverse shock. The high temperature and density implies that thermal conduction by electrons may be an important process for the evolution of dense ejecta knots, with implications for the survival of supernova dust.

Second, CO observations have been used to study the circumstellar environment of a massive star, the yellow hypergiant IRAS 17163-3907. Our observations with APEX (Paper II) and ALMA ACA (Paper III) reveal a complex environment with several distinct components: a fast recent stellar wind of $100 \mathrm{~km} \mathrm{~s}^{-1}$, a clumpy CO ring which appears to be a torus ejected by the star several thousand years ago, and a unidirectional bright spur extending from the star to the clumpy ring. These asymmetries are not seen in infrared dust observations, and demonstrate the complexity of massive evolved stars and the need for high resolution molecular observations to understand them.

Finally, the contribution of massive stars to galactic chemical enrichment has been investigated indirectly with measurements of isotopic ratios in a molecular absorber at $\mathrm{z}=0.68$ towards $\mathrm{B} 0218-211$ (Paper IV). We find that the ratios at $\mathrm{z}=0.68$ are very different from those in the solar neighborhood, but similar to the ratios found in another absorber at $\mathrm{z}=0.89$ and in starburst galaxies. We interpret these ratios as the signature of enrichment mainly by massive stars.

From the evolution of a supernova remnant, to the complex surroundings of a massive evolved star, to the isotopic enrichment of a distant galaxy, this thesis addresses the role massive stars play in shaping and enriching their environments. Massive stars may not be numerous, but their death throes echo across time and space.
\end{abstract}

Keywords: submillimeter: ISM - ISM: supernova remnants - ISM: individual objects: Cassiopeia A - circumstellar matter - stars: AGB and post-AGB - stars: mass-loss stars: individual: IRAS 17163-3907 - quasars: absorption lines - quasars: individual: B 0218+357 - galaxies: ISM - galaxies: abundances - ISM: molecules 


\section{Research contributions}

This thesis is based on the work contained in the following papers:

I. S. H. J. Wallström, C. Biscaro, F. Salgado, J. H. Black, I. Cherchneff, S. Muller, O. Berné, J. Rho \& A. G. G. M. Tielens :

CO rotational line emission from a dense knot in Cassiopeia A: Evidence for active post-reverse-shock chemistry

Astronomy \& Astrophysics, 558, 2 (2013)

II. S. H. J. Wallström, S. Muller, E. Lagadec, J. H. Black, K. Justtanont, R. Oudmaijer, H. van Winckel \& A. Zijlstra:

Investigating the nature of the Fried Egg nebula: $\mathrm{CO}$ mm-line and optical spectroscopy of IRAS 17163-3907

Astronomy \& Astrophysics, 574, 139 (2015)

III. S. H. J. Wallström, E. Lagadec, S. Muller, J. H. Black, N. L. J. Cox, R. Galván-Madrid, K. Justtanont, S. Longmore, H. Olofsson, R. D. Oudmaijer, G. Quintana-Lacaci, R. Szczerba, W. Vlemmings, H. van Winckel \& A. Zijlstra:

ALMA Compact Array observations of the Fried Egg nebula: Evidence for largescale asymmetric mass-loss from the yellow hypergiant IRAS 17163-3907

Submitted to A\&A

IV. S. H. J. Wallström, S. Muller \& M. Guélin

Isotopic ratios at $z=0.68$ from molecular absorption lines towards B $0218+357$

Submitted to A\&A

I have also contributed some optical observations to the following paper, which is not discussed in the thesis:

- R. Amanullah, J. Johansson, A. Goobar, R. Ferretti, S. Papadogiannakis, T. Petrushevska, P. J. Brown, Y. Cao, C. Contreras, H. Dahle, N. Elias-Rosa, J. P. U. Fynbo, J. Gorosabel, L. Guaita, L. Hangard, D. A. Howell, E. Y. Hsiao, E. Kankare, M. Kasliwal, G. Leloudas, P. Lundqvist, S. Mattila, P. Nugent, M. M. Phillips, A. Sandberg, V. Stanishev, M. Sullivan, F. Taddia, G. Östlin, S. Asadi, R. Herrero-Illana, J. J. Jensen, K. Karhunen, S. Lazarevic, E. Varenius, P. Santos, S. Seethapuram Sridhar, S. H. J. Wallström \& J. Wiegert:

Diversity in extinction laws of Type Ia supernovae measured between 0.2 and 2 $\mu \mathrm{m}$

Monthly Notices of the Royal Astronomical Society, 453, 3300 (2015) 


\section{Acknowledgements}

A big thank you to my supervisors, Sébastien Muller and John Black, for their wisdom, encouragement, and patience. Thank you to everyone at the observatory, especially my fellow PhD students, for making it a lovely place to work, and thank you to the space bus for getting us there.

Thank you to all my collaborators, for sharing their knowledge and broadening mine.

And thank you to my family, and Shane, for their unwavering support.

Sofia 



\section{Contents}

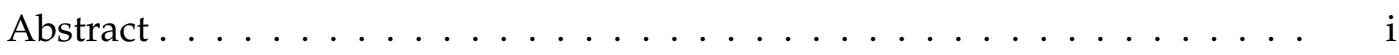

Research contributions ................... iii

Acknowledgements .......................

1 Introduction 1

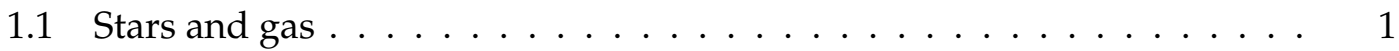

1.1.1 Stellar lifecycle . . . . . . . . . . . . . . . . . 2

1.1.2 Stellar nucleosynthesis . . . . . . . . . . . . . . . 3

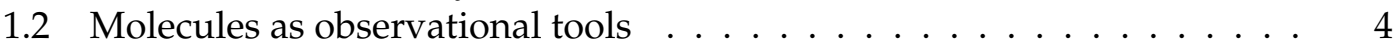

1.2.1 Molecular emission . . . . . . . . . . . . . . . . . 5

1.2.2 Molecular absorption . . . . . . . . . . . . . . 8

1.2.3 Rotation diagrams . . . . . . . . . . . . . . . . . 8

1.2 .4 RADEX . . . . . . . . . . . . . . . . . . . . . 10

1.2 .5 Telescopes . . . . . . . . . . . . . . . . . . . 10

2 Supernova remnants $\mathbf{1 5}$

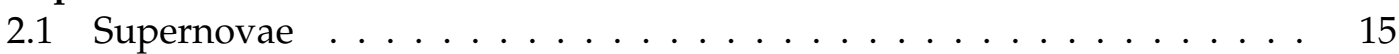

2.1 .1 SN 1987A . . . . . . . . . . . . . . . . . . . 16

2.1.2 Molecules and dust in supernova ejecta . . . . . . . . . . . . . 17

2.1 .3 Cassiopeia A . . . . . . . . . . . . . . . 17

2.2 Introduction to Paper I . . . . . . . . . . . . . . . . . . . . 18

2.2 .1 Heating mechanisms . . . . . . . . . . . . . . . . 19

2.3 Future prospects . . . . . . . . . . . . . . . . . 21

3 Massive evolved stars 23

3.1 Yellow Hypergiants . . . . . . . . . . . . . . . . . . . . . . 23

3.1 .1 IRAS $17163-3907 \ldots \ldots \ldots \ldots \ldots$

3.2 Introduction to Paper II . . . . . . . . . . . . . . . . . . . . . . 27

3.3 Introduction to Paper III . . . . . . . . . . . . . . . . . . . . . 27

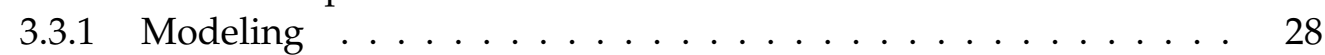

3.4 Future prospects . . . . . . . . . . . . . . . . . . . 29

vii 
4 Galactic chemical evolution 31

4.1 Chemical evolution models . . . . . . . . . . . . . . . . . 32

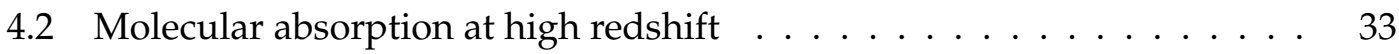

4.2.1 The $\mathrm{z}=0.89$ absorber towards PKS $1830-211 \ldots \ldots$. . . . . . . 34

4.2.2 The $\mathrm{z}=0.68$ absorber towards B $0218+357 \ldots \ldots$. . . . . . . 35

4.3 Introduction to Paper IV . . . . . . . . . . . . . . 36

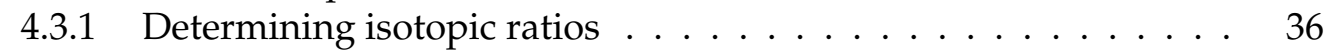

4.4 Future prospects . . . . . . . . . . . . . . . 36

$\begin{array}{ll}\text { Bibliography } & 39\end{array}$

$\begin{array}{ll}\text { Paper I } & 43\end{array}$

$\begin{array}{ll}\text { Paper II } & 51\end{array}$

$\begin{array}{lr}\text { Paper III } & 69\end{array}$

$\begin{array}{ll}\text { Paper IV } & 81\end{array}$ 


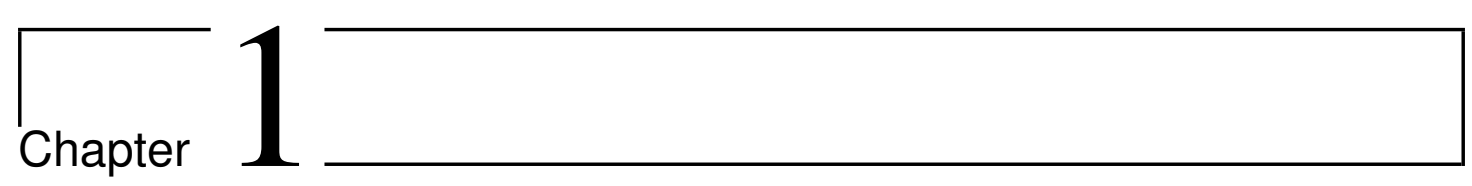

\section{Introduction}

\subsection{Stars and gas}

Our cultural perception of the Universe is full of stars, from the constellations in the night sky to glittering images of star-filled galaxies. It's not a complete picture of the Universe, but it is a good starting point given that stars are essentially the little engines of our galaxy. Stars provide energy and ionizing radiation to the surrounding gas of the interstellar medium (ISM), and, more importantly, stars have created every atom heavier than hydrogen and helium (what astronomers call metals) that exists in the Universe.

Stars gain their energy from nucleosynthesis in their cores, fusing lighter atoms to form heavier ones. A fraction of these metals are then expelled into the ISM via stellar winds. The most massive stars will eventually explode as supernovae, creating even heavier elements via explosive nucleosynthesis. The ejected material, replete with metals, will expand and eventually integrate into the ISM. In this way, stars drive the chemical evolution of galaxies as they add more and more metals to the interstellar gas.

From metals, a wide variety of molecules and dust grains can form, which provide important cooling mechanisms for the gas and affect its chemistry. Molecular transitions are a main cooling mechanism at low temperatures, allowing dense molecular clouds to cool, fragment, and collapse to form stars. Without molecules this process would be much less efficient, and incapable of forming low-mass stars.

Molecules are also useful observationally, as tracers of gas. By observing molecular transitions we can probe a wide variety of gas phases, from stellar winds to supernova remnants, and study gas kinematics, excitation, and chemistry. Molecular astronomy is an important tool for studying various stages of stellar life, from birth to death. 


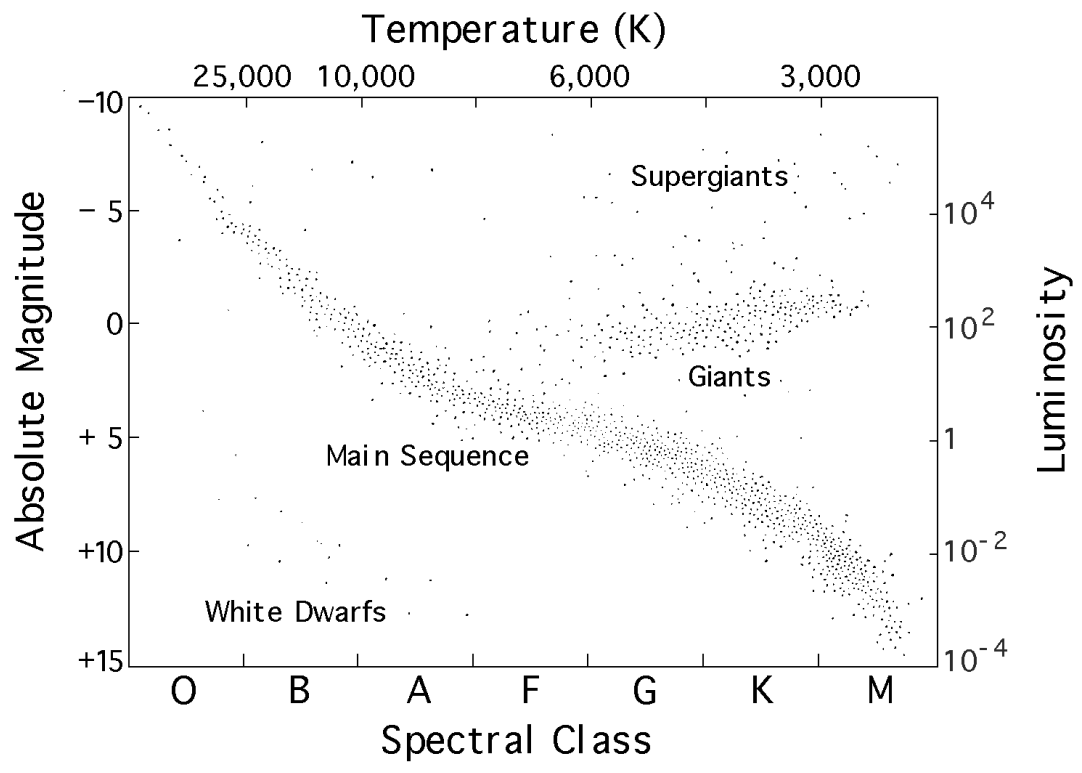

Figure 1.1: A Hertzsprung-Russell diagram of nearby stars. Luminosity is given in solar luminosities, $\mathrm{L}_{\odot}$, equal to $3.828 \times 10^{26} \mathrm{~W}$. (Image credit: $N A S A / H E A S A R C$ )

\subsubsection{Stellar lifecycle}

Stars are born from the collapse of dense molecular clouds, gathering gas until they are heavy and hot enough to ignite nuclear fusion. Stars can be born with a wide range of masses, in proportions approximated by the Salpeter initial mass function (IMF): $N(m) \propto m^{-2.35}$, where $N(m)$ is the number of stars of mass $m$ (Salpeter, 1955). This means that low-mass stars are significantly more numerous than high-mass stars. For example, there are about 225 times as many solar-mass $\left(1 \mathrm{M}_{\odot}\right)$ stars as there are stars of mass $10 \mathrm{M}_{\odot}$. Mass is an important factor in determining the properties of stars, along with their surface temperatures and luminosities.

In order to classify stars, one can look at their locations in the HertzsprungRussell (HR) diagram (Figure 1.1). This is a plot of luminosity against temperature, and a star's location on the HR diagram indicates both its mass and evolutionary status. Most stars are on the "main sequence", which corresponds to the main, hydrogen-burning part of a star's life. Stars with high temperatures and luminosities (high-mass stars) are on the blue (left) end of the main sequence, while stars with low temperatures and luminosities (low-mass stars) are on the red (right) end. After a star has depleted the hydrogen in its core it contracts, heating up until the core is hot enough to start burning helium, which only happens for stars of mass $>0.5 \mathrm{M}_{\odot}$. The hot helium-burning core causes the surrounding layers to inflate, so the star moves off the main sequence and 


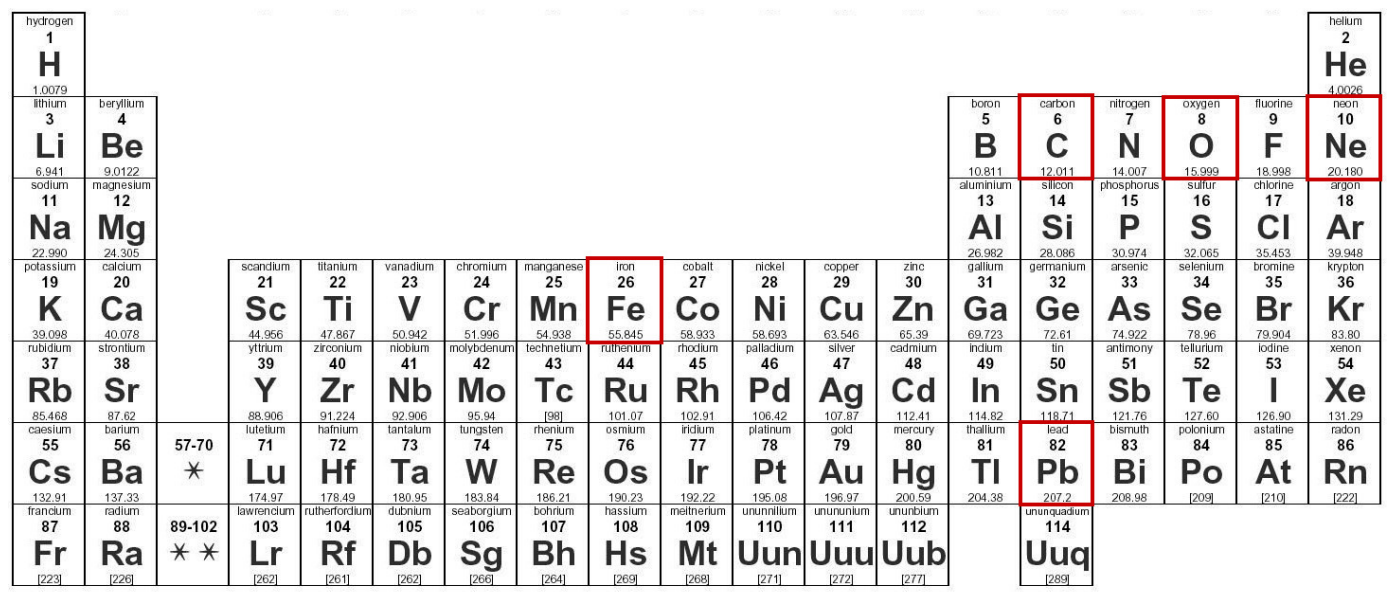

\begin{tabular}{|c|c|c|c|c|c|c|c|c|c|c|c|c|c|c|}
\hline \multirow{2}{*}{ * Lanthanide series } & 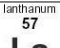 & 58 & D & N & 61 & 62 & 63 & ${ }^{64}$ & 65 & 66 & $\begin{array}{l}\text { nolmum } \\
67\end{array}$ & 68 & 69 & $7^{70}$ \\
\hline & La & $\mathrm{Ce}$ & $\mathrm{Pr}$ & $\mathrm{Nd}$ & Pm & Sm & Eu & Gd & $\mathrm{Tb}$ & Dy & Ho & Er & Tm & Yb \\
\hline \multirow[t]{2}{*}{${ }^{*}$ Actinide series } & $\begin{array}{l}\text { actinum } \\
89\end{array}$ & $\begin{array}{l}\text { throrium } \\
90\end{array}$ & 91 & 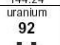 & $\begin{array}{l}\text { neptunum } \\
\text { epunum }\end{array}$ & $\begin{array}{l}\text { llojiom } \\
\text { putonum }\end{array}$ & $\begin{array}{l}\text { Miriciom } \\
\text { arcicum }\end{array}$ & 96 & 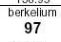 & 98 & 99 & $\begin{array}{c}\text { terti } \\
\text { tetmim } \\
100\end{array}$ & 101 & netion \\
\hline & Ac & Th & $\mathbf{P a}$ & $\mathrm{U}$ & $\mathrm{Np}$ & $\mathrm{Pu}$ & Am & $\mathrm{Cm}$ & Bk & Cf & Es & Fm & Md & No \\
\hline
\end{tabular}

Figure 1.2: Periodic table of elements. Some important elements, mentioned in the text below, are highlighted in red. (c) User:LeVanHan/Wikimedia Commons/CC-BY-SA-3.0)

up in the HR diagram towards larger luminosities. This will happen sooner the more massive the star is: the Sun has a main sequence lifetime of about 10 billion years, compared with only $\sim 30$ million years for a $10 \mathrm{M}_{\odot}$ star. There are several tracks a star may follow in the HR diagram, depending on its physical parameters, but generally low-mass stars will come around to the white dwarf branch while high-mass stars instead end their lives as supernovae.

\subsubsection{Stellar nucleosynthesis}

A few minutes after the Big Bang, the Universe had cooled enough for the first atoms to form: mainly hydrogen, plus about $25 \%$ helium (by mass) and trace amounts of lithium and beryllium. All other elements are formed by nucleosynthesis in stars, either as part of their energy generation as they live or in the spectacular explosions as they die. The smallest stars, with masses less than half a solar mass $\left(<0.5 \mathrm{M}_{\odot}\right)$, will fuse hydrogen into helium but never get hot enough for helium fusion and hence will produce no metals. Heavier stars, up to $8 \mathrm{M}_{\odot}$, will be able to create carbon $(\mathrm{C})$ and oxygen $(\mathrm{O})$ in their cores. It takes massive stars, $>8 \mathrm{M}_{\odot}$, to create neon $(\mathrm{Ne})$ and other heavy elements up to iron (Fe, atomic number 26 - see Figure 1.2), which is the heaviest element to be produced by stable nuclear fusion. All stars with masses $>1 \mathrm{M}_{\odot}$ can also create some heavy elements through the slow neutron capture process (s-process). 
Reactions of the isotopes carbon-13 and neon-22 with helium produce free neutrons, which can be captured by other atoms to create heavier elements. These atoms have time to $\beta$-decay to a stable state before potentially capturing another neutron, hence the name slow neutron capture. The s-process produces elements up to lead $(\mathrm{Pb}$, atomic number 82$)$, with peaks in abundance around especially stable elements like strontium ( $\mathrm{Sr}$ ) and barium $(\mathrm{Ba})$. The creation of elements heavier than lead requires the rapid neutron capture process ( $r$ process), where a high neutron flux allows atoms to capture several neutrons without decaying to a stable state in between captures. The r-process is only found in the explosive nucleosynthesis of supernovae, which also expel large amounts of lighter elements.

\subsection{Molecules as observational tools}

Molecules are an important tool for probing cold/cool gas $(\mathrm{T}<100 \mathrm{~K})$. They are fairly easily excited and emit radiation at radio frequencies, which can penetrate the atmosphere, and are hence observable from the Earth (see $\S 1.2 .5$ and Figure 1.5). Radio frequencies also allow for good velocity resolution, through the use of heterodyne spectrometers with resolving power, $\mathrm{R}=\nu / \Delta \nu$, of up to $10^{7}$, corresponding to a velocity resolution $(\Delta \mathrm{v}=\mathrm{c} \Delta \nu / \nu)$ of $0.03 \mathrm{~km} \mathrm{~s}^{-1}$. Hence, molecular lines allow us to study gas kinematics in high detail, and the line excitation probes the physical conditions of the gas. Observing several different molecules can also elucidate the gas chemistry, helping to constrain astrochemical models. Furthermore, different isotopologues of the same molecule tend to be well separated in frequency, and hence can be used to derive isotopic ratios.

Molecular gas makes up about 20\% of the mass of the ISM (Tielens 2005), and the most common molecule by far is $\mathrm{H}_{2}$, since most atoms in the Universe are hydrogen. As $\mathrm{H}_{2}$ is a symmetric molecule it has zero dipole moment and hence no allowed rotational transitions, so it is not easily detected in cold gas (see $\S 1.2 .1$. Instead we rely on tracer molecules to study the ISM. Almost 200 molecules have been detected in the ISM and circumstellar material, so far ${ }^{\mathrm{a}}$, but one of the most observationally useful is carbon monoxide (CO).

$\mathrm{CO}$ is the second most common molecule in the Universe, with an abundance of $\sim 10^{-4}$ (relative to $\mathrm{H}_{2}$ ) in the ISM. It forms readily, and is very stable due to the triple bond between the carbon and oxygen atoms. $\mathrm{CO}$ is a heterogeneous diatomic molecule, with a dipole moment of $0.122 \mathrm{D}$, so it has a range of low-frequency rotational transitions (Figure 1.3). The dipole moment of CO is small in absolute terms, and hence the emission lines are not as intrinsically strong as the lines of more polar molecules, but the large $\mathrm{CO}$ abundance (orders of magnitude greater than other ISM molecules) overcomes this and CO lines

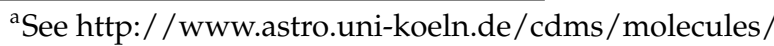




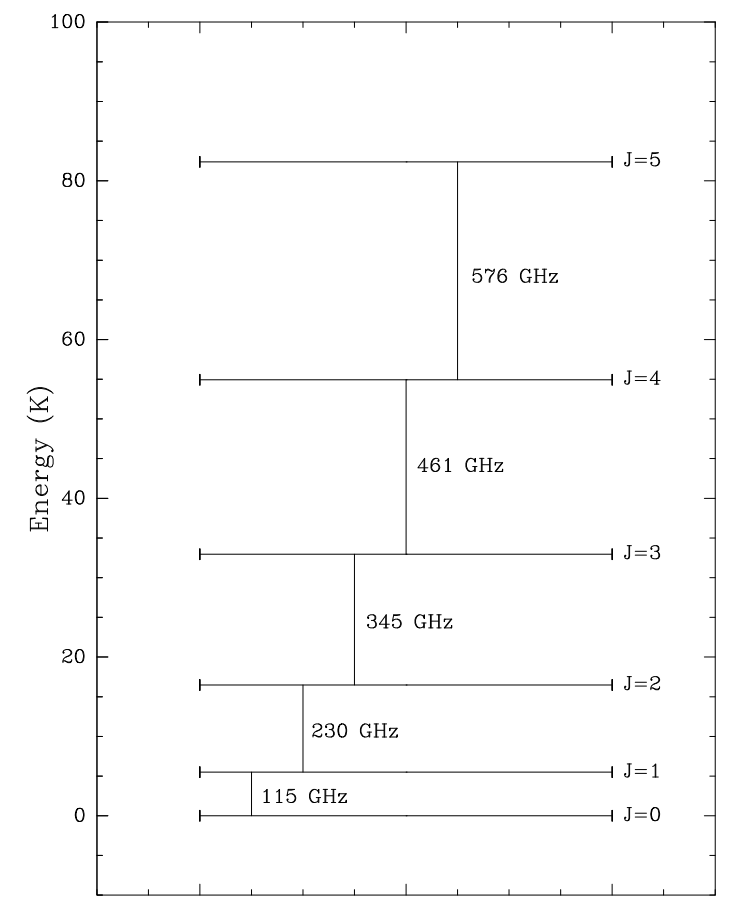

Figure 1.3: First few pure rotational energy levels of $\mathrm{CO}$, and the transition frequencies.

are readily observable. The lowest transitions are easily excited in cold ISM gas $\left(E_{u p}<30 \mathrm{~K}\right.$, see Figure 1.3), allowing us to study, e.g., Giant Molecular Clouds, while the higher transitions trace warmer, denser gas, such as might be found in shocks around stars. $\mathrm{CO}$ also has several common isotopologues (e.g., ${ }^{13} \mathrm{CO}$, $\mathrm{C}^{18} \mathrm{O}, \mathrm{C}^{17} \mathrm{O}$ ) that can be used to probe large column densities of gas which are optically thick in lines of the main ${ }^{12} \mathrm{C}^{16} \mathrm{O}$ isotopologue.

\subsubsection{Molecular emission}

General reference: Wilson (2009)

As a consequence of quantum mechanics, the energy levels of molecules are discrete. Transitions between these levels result in the emission of electromagnetic radiation at specific frequencies, the observation of which lets us deduce something about the physical conditions of the molecule. There are three types of molecular emission: electronic, vibrational, and rotational.

Electronic emission involves the transitions of electrons between energy levels. These transitions have typical energies of a few electron volts $(\mathrm{eV})$, which corresponds to radiation in the visible and UV parts of the electromagnetic spectrum, with wavelengths around $100 \mathrm{~nm}$. Vibrational emission involves the 
bonds between the atoms stretching and bending, and is mainly in the infrared (IR) part of the spectrum, with wavelengths of several $\mu \mathrm{m}$. Rotational emission is due to transitions between the quantised rotational states of the molecule and is seen in the radio/sub-mm part of the spectrum, with typical wavelengths around $1 \mathrm{~mm}$.

For illustration, the simple case of rotation in a linear diatomic molecule (with zero net electron spin) can be mathematically described as follows: a linear molecule can be approximated as a rigid rotor, so the energy of rotational state $\mathrm{J}$ above the ground state is given by

$$
E=h B_{0} J(J+1)
$$

where $J$ is the angular momentum quantum number, $h$ is the Planck constant, and $B_{0}$ is the rotational constant. Only rotational transitions with $\Delta J=1$ are allowed by the quantum mechanical selection rules. The corresponding transition frequencies are approximately $\nu=\Delta E / h=2 B_{0}(J+1)$. The state energy is associated with a temperature, given by $E / k_{B}$, in Kelvin.

The probability for a molecule to spontaneously transition from the $\mathrm{J}+1$ to $\mathrm{J}$ state $(u$ to $l$ ) is given by the Einstein A coefficient

$$
A_{u l}=\frac{64 \pi^{4}}{3 h c^{3}} \nu^{3}\left|\mu_{u l}\right|^{2}
$$

where $A_{u l}$ is in $\mathrm{s}^{-1}$, and $\mu_{u l}$ is the dipole moment, which is the product of charge and the separation of charges and hence analogous to classical angular momentum. The dipole moment $\left|\mu_{u l}\right|^{2}$ can be expressed by its radial part $\mu$ as:

$$
\left|\mu_{J+1 \rightarrow J}\right|^{2}=\mu^{2} \frac{J+1}{2 J+3}
$$

giving

$$
A_{u l} \simeq 1.165 \times 10^{-11} \mu^{2} \nu^{3} \frac{J+1}{2 J+3}
$$

where $\nu$ is the transition frequency in $\mathrm{GHz}$, and $\mu$ is in Debye.

The Einstein A coefficient gives the probability for the upper level to decay to the lower level by spontaneous emission of a photon. Hence, the inverse gives an estimate of the lifetime of the upper state. Typical values of $A_{u l}$ for interstellar molecules are $10^{-8}-10^{-3} \mathrm{~s}^{-1}$, corresponding to upper level lifetimes ranging from hours to years.

In order for a molecule to emit radiation by decaying to a lower level, it must first be excited to an upper level. Molecules can be excited by absorbing radiation or from collisions with surrounding particles (most likely $\mathrm{H}_{2}$ ). The main excitation mechanism for a given molecule depends on the structure of the molecule itself, e.g., the energy level spacing, as well as its surroundings: 
the density of the surrounding gas and the strength of the background radiation field.

In a two level system, consisting of an upper level $u$ and a lower level $l$, the populations of the levels are set by the rates of collision, absorption, and spontaneous and stimulated emission. At statistical equilibrium $\left(\mathrm{d} n_{i} / \mathrm{d} t=0\right)$, in the absence of chemical sources or sinks:

$$
\begin{gathered}
\frac{n_{u}}{n_{l}}=\frac{n q_{l u}+B_{l u} I}{n q_{u l}+A_{u l}+B_{u l} I} \\
A_{u l}=\frac{8 \pi h \nu^{3}}{c^{3}} B_{u l}
\end{gathered}
$$

with $n$ the gas density, and $I$ the radiation density. $q_{i j}$ are the collisional coefficients, and $B_{i j}$ the stimulated emission/absorption coefficients.

For a more intuitive understanding, we can define two temperatures, $T_{e x}$ and $T_{k}$, by

$$
\begin{gathered}
\frac{q_{l u}}{q_{u l}}=\frac{g_{u}}{g_{l}} \exp \left(-\frac{T_{0}}{T_{k}}\right), \text { with } T_{0} \equiv \frac{h \nu}{k_{B}} \\
\frac{n_{u}}{n_{l}}=\frac{g_{u}}{g_{l}} \exp \left(-\frac{T_{0}}{T_{e x}}\right)
\end{gathered}
$$

The kinetic temperature $T_{k}$ is the physical temperature of the gas. The excitation temperature $T_{e x}$ is the temperature at which we would expect to find this ratio of level populations, according to a Boltzmann distribution. Note that $T_{e x}$ is not necessarily a physical temperature: it can take a wide range of values and can even be negative when level populations are inverted, as in masers.

In radio astronomy, we also define another temperature for the intensity of the emission. For a brightness $I$ in units of $\mathrm{W} \mathrm{m}^{-2} \mathrm{~Hz}^{-1} \mathrm{sr}^{-1}$, the brightness temperature, $T_{b}$ is defined as

$$
T_{b}=\frac{\lambda^{2}}{2 k_{B}} I
$$

which is the temperature of a black body of brightness $I$, in the Rayleigh-Jeans approximation (when $h \nu \ll k_{B} T$, where $k_{B}$ is the Boltzmann constant) which applies well to emission with wavelengths on the order of meters to centimeters.

In general, $T_{k}, T_{e x}$, and $T_{b}$ can be different. Substituting these quantities into equation 1.5 we find an expression for $T_{e x}$ :

$$
T_{e x}=T_{k} \frac{T_{b} A_{u l}+T_{0} n q_{u l}}{T_{k} A_{u l}+T_{0} n q_{u l}}
$$

If collisions dominate the excitation, i.e., if $n q_{u l} \gg A_{u l}, T_{e x}$ tends towards $T_{k}$. Under these conditions the gas is in local thermal equilibrium (LTE), and 
can be defined by a single equilibrium temperature, equal to $T_{e x}=T_{k}$. If radiation dominates the excitation, and $n q_{u l} \ll A_{u l}, T_{e x}$ will instead tend towards $T_{b}$. The density at which collisions and radiation have roughly equal contributions is called the critical density, $n_{c} \simeq A_{u l} / q_{u l}$. As $A_{u l}$ depends on the transition frequency, different transitions of the same molecule will have different $n_{c}$, increasing with $J$. In general, if an environment satisfies $n>n_{c}$ and $T>E_{u} / k_{B}$ for a given molecular transition, we expect that transition to be excited. Whether we can detect this emission, however, depends on further factors, including the abundance of the molecule. Note also that the above discussion is limited by the assumption of a two-level system.

\subsubsection{Molecular absorption}

\section{General reference: Combes $\mathcal{E}$ Wiklind (1996)}

Molecules will also absorb electromagnetic radiation at the same frequencies they emit it. So, given a sufficiently bright background continuum source, absorption lines can be used to study interstellar gas. This method has several advantages. First, the detectability of the molecular lines depends only on the observed flux of the background source, as they are seen in absorption against it. It is therefore not affected by distance, meaning that very distant galaxies and faint lines can be probed. In addition, provided the background source is completely covered by the absorbing gas, the absorption depth is proportional to the line opacity for optically thin lines. This allows for direct comparison between different lines and hence the calculation of, e.g., abundances or isotopic ratios.

However, this method is limited by the need for a chance alignment of a bright background source and intervening ISM, which means there are few candidate sources. It is also biased towards the detection of low-J transitions of molecules with a strong dipole moment. This corresponds to a bias towards diffuse gas, where there are insufficient collisions to populate the higher energy levels. Therefore, extra care must be taken when comparing sources observed in absorption with those observed in molecular emission lines, as emission lines are often more easily observed in dense gas.

\subsubsection{Rotation diagrams}

As discussed in $\S 1.2 .1$, at densities greater than the critical density, $n_{c}$, we can assume the emitting gas can be described by a single excitation temperature: $T_{e x}=$ $T_{k}$. If we have observations of more than one transition of the same molecule, we can derive this temperature and the total column density (molecules per unit area) of the molecule. This is done by creating a rotation diagram, as described 


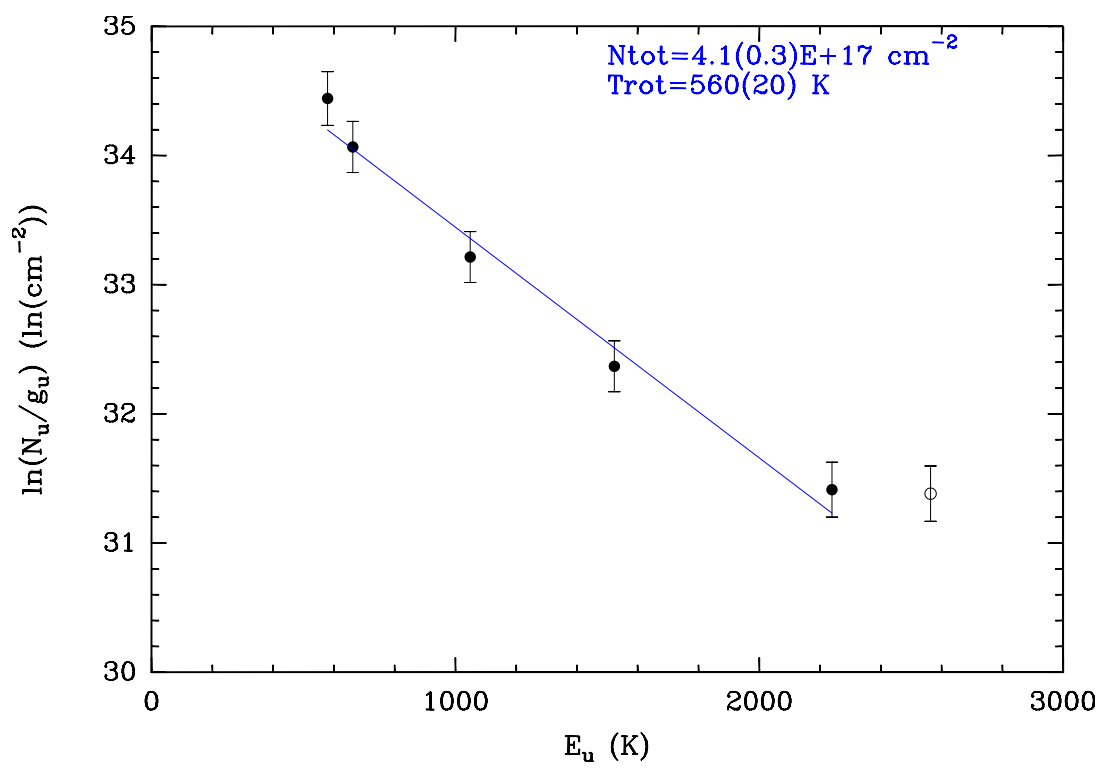

Figure 1.4: Rotation diagram from Paper I.

by Goldsmith \& Langer (1999) and summarized below. An example rotation diagram, from Paper I, is shown in Figure 1.4.

It can be shown that the integrated intensity, $I_{u l}$, of an optically thin emission line is related to the population density of the upper energy level, $N_{u}$, by

$$
\int I_{u l}=\frac{N_{u} A_{u l} h \nu_{u l}}{4 \pi}
$$

where $A_{u l}$ is the Einstein coefficient for the $u \rightarrow l$ transition. Hence we can derive $N_{u}$ from line observations.

In LTE, the energy level populations follow a Boltzmann distribution, so that

$$
N_{u}=\frac{N}{Z} g_{u} e^{-E_{u} / T_{e x}}
$$

where $N$ is the total column density, $Z$ is the partition function, $g_{u}$ is the statistical weight of the upper level $u$, and $E_{u}$ is the energy of the upper level in Kelvin.

If we take the logarithm of this equation we get a simple linear relation between $\ln N_{u} / g_{u}$ and $E_{u}$ :

$$
\ln \frac{N_{u}}{g_{u}}=\ln \frac{N}{Z}-E_{u} / T_{e x}
$$

Plotting this relation for all observed transitions of a molecule will allow us to derive the excitation temperature, $T_{e x}$, from the slope of the line, and the 
total column density of the molecule, $N$, from the y-intercept. This method is fast and straightforward (if the emission lines are optically thin), but it does rely on the assumption that the gas an ideal two-level system in LTE.

\subsubsection{RADEX}

There are ways of determining the temperature and density of a molecule without the assumption of a constant excitation temperature for all levels, and one of the most straightforward is implemented in the freely available RADEX ${ }^{\mathrm{b}}$ program (van der Tak et al., 2007). RADEX calculates the non-equilibrium excitation levels of a molecule by assuming a uniform emitting region (i.e., constant density, temperature, and abundance). It requires the collisional rates between the molecule and a collision partner (usually $\mathrm{H}_{2}$, but can also be, e.g., $\mathrm{H}_{2} \mathrm{O}$ or electrons), which are available for some 40 different molecules in the LAMDA ${ }^{c}$ molecular database, including many commonly observed molecules such as $\mathrm{CO}, \mathrm{HCN}$, and $\mathrm{H}_{2} \mathrm{O}$.

RADEX takes as input parameters the density of the collision partner, and the column density and kinetic temperature of the molecule. It then iteratively solves for the level populations and the radiation field, stopping when the calculated optical depths are stable from one iteration to the next. By testing a range of input parameters, one can fit the line strengths predicted by RADEX to observed data, thereby constraining the density, column density, and kinetic temperature of the gas without an assumption of LTE. This is extremely useful as an approximation of the physical parameters of an observed molecule.

\subsubsection{Telescopes}

In order to observe the emission or absorption from interstellar molecules, we need large and powerful telescopes. The diameter of a single dish telescope, $D$, determines its sensitivity $\left(\propto D^{2}\right)$ and angular resolution $(\sim \lambda / D)$, so as large a telescope as possible is the ideal. However, the required surface accuracy of the dish is a fraction of the wavelength of the observed radiation, so as we aim for higher energy transitions at higher frequencies (shorter wavelengths), the maximum dish size is limited.

One way around this problem is to use the technique of radio interferometry. Interferometry correlates the data from multiple antennae to "synthesize" a single dish as large as the maximum distance between the antennae (called the baseline). This is a powerful technique, allowing us to reach resolutions of less than a milliarcsecond, though the complicated image synthesis means that data interpretation can be difficult.

\footnotetext{
${ }^{b}$ http:/ / www.sron.rug.nl/ vdtak/radex/radex.php

chttp://home.strw.leidenuniv.nl/ moldata/molecules.html
} 


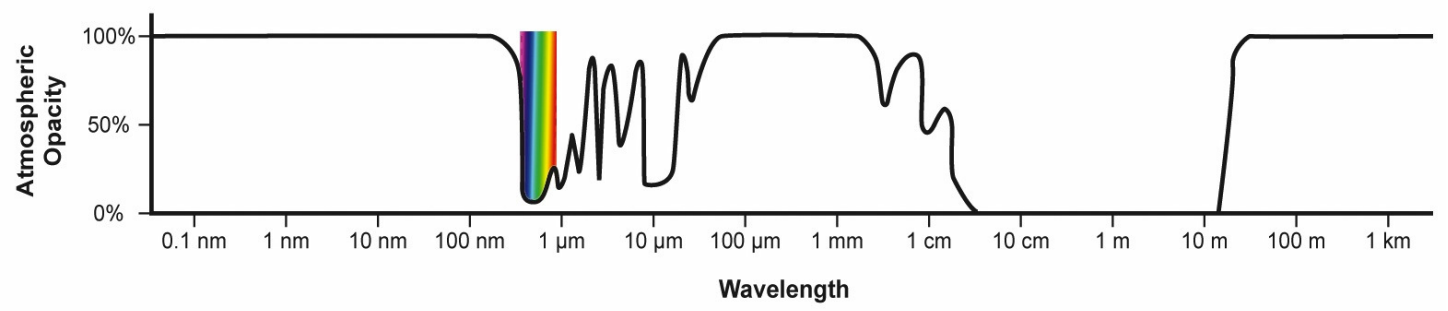

Figure 1.5: The opacity of the atmosphere at different wavelengths. Most radiation is absorbed completely (opacity 100\%) outside the windows at optical/near-IR $(\sim 1 \mu \mathrm{m})$ and radio $(1 \mathrm{~cm}-10 \mathrm{~m})$ wavelengths. (Image credit: NASA/IPAC)

Another observational challenge is the Earth's atmosphere. The atmosphere absorbs most of the radiation that hits it, though there are transmission "windows" in the optical/near-IR and radio parts of the spectrum (Figure 1.5), which allow radiation through. Furthermore, fluctuations in the atmosphere distort observations, even in the atmospheric windows. To circumvent the problems of the atmosphere, telescopes can either be put on mountains, above as much of the atmosphere as possible, or in space.

For observing pure rotational $\mathrm{CO}$ emission, the required wavelengths range between $\sim 1 \mathrm{~mm}$ and $100 \mu \mathrm{m}$. At these wavelengths the atmosphere's opacity is strongly affected by water vapor, and the effect increases with shorter wavelengths. Around $100 \mu \mathrm{m}$ the atmosphere is completely opaque. Hence, low- $\mathrm{J}$ CO transitions $(\mathrm{J}<10)$ require a telescope site that is both high and dry, while high-J transitions require a space telescope. Some examples of $\mathrm{mm} / \mathrm{submm}$ telescopes relevant to this thesis are presented below.

\section{Herschel}

The Herschel Space Observatory ${ }^{\mathrm{d}}$ was launched into orbit in May 2009, as part of the European Space Agency (ESA) science programme, and ceased operations in April 2013. It is a 3.5m telescope, the largest ever flown in space, with imaging and spectroscopic instruments operating in the $55-671 \mu \mathrm{m}$ infrared wavelength range. The data for Paper I were obtained with Herschel, using the Photodetector Array Camera and Spectrometer (PACS) instrument (Poglitsch et al., 2010). PACS has a field of view of $\sim 50^{\prime} \times 50^{\prime}$, divided into a $5 \times 5$ array of pixels. We observed several CO transitions in the wavelength range $87-186 \mu \mathrm{m}$.

\footnotetext{
${ }^{\mathrm{d}}$ http:/ / www.esa.int/herschel
} 


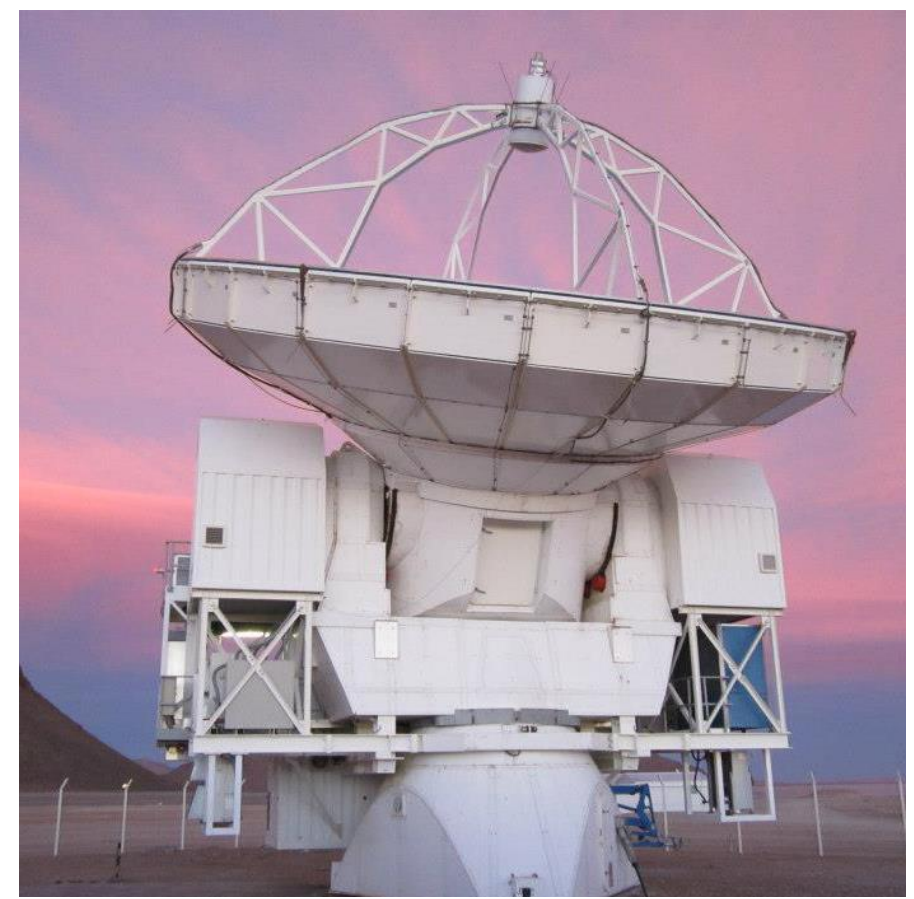

Figure 1.6: A photo of the APEX telescope (Image credit: Sofia Wallström)

\section{APEX}

The Atacama Pathfinder EXperiment (APEXe, shown in Figure 1.6) is a $12 \mathrm{~m}$ $\mathrm{mm} / \mathrm{submm}$ telescope located at Llano de Chajnantor in the Atacama desert in northern Chile, at an elevation of $5100 \mathrm{~m}$. It is operated jointly by the Onsala Space Observatory (OSO), Max Planck Institut für Radioastronomie (MPIfR) and the European Southern Observatory (ESO). The high altitude and dry and stable atmosphere makes the location ideal for a $\mathrm{mm} / \mathrm{submm}$ telescope. The CO data for Paper II were obtained with APEX, with the heterodyne receivers at 230 and $345 \mathrm{GHz}$ (corresponding to wavelengths around $1 \mathrm{~mm}$ ).

\section{ALMA}

The Atacama Large Millimeter/submillimeter Array $\left(\mathrm{ALMA}^{\mathrm{f}}\right)$ is an interferometer consisting of $5412 \mathrm{~m}$ antennas and $127 \mathrm{~m}$ antennas, located at Llano de Chajnantor in Chile, next to APEX. It is a large international collaboration between Europe, North America, East Asia, and Chile. ALMA has excellent sensitivity ( $<10 \mu \mathrm{Jy} /$ beam $)$, and maximum baselines of up to $16 \mathrm{~km}$ which allows for

\footnotetext{
ehttp:/ / www.apex-telescope.org/

${ }^{\mathrm{f}}$ http:/ / www.almaobservatory.org/
} 


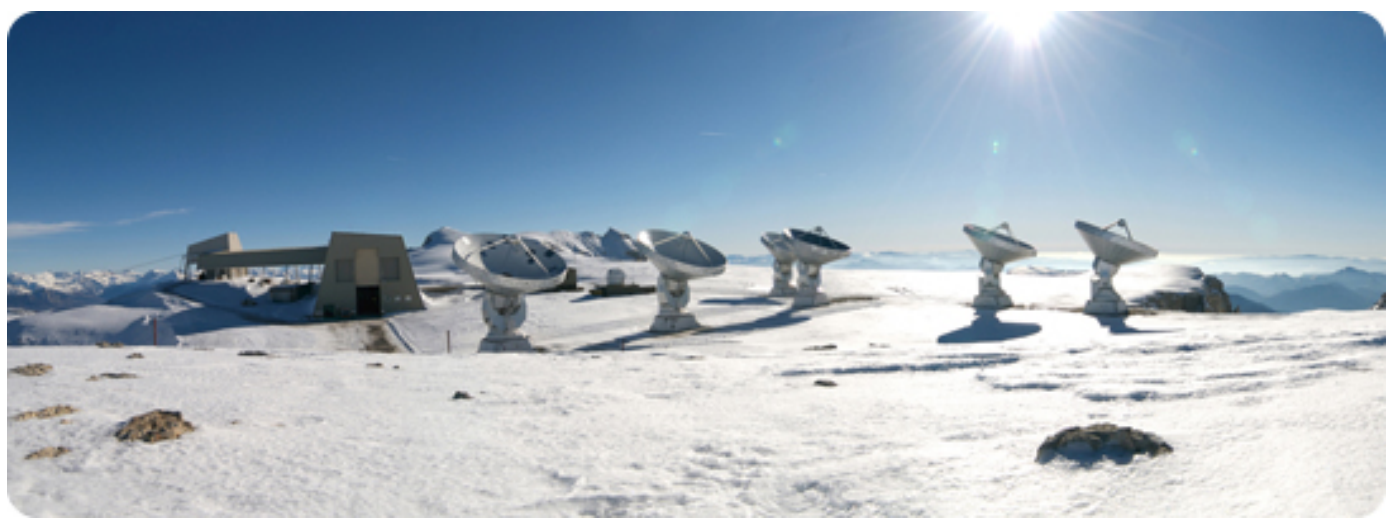

Figure 1.7: A photo of the PdBI telescope (Image credit: IRAM)

very high angular resolution (0.006"). Paper III uses data from the ALMA Compact Array (ACA), the closely spaced set of $7 \mathrm{~m}$ dishes used to provide short baseline data. Our $\mathrm{CO}$ data at $230 \mathrm{GHz}$ have an angular resolution of about 8 ".

\section{PdBI}

The Plateau de Bure Interferometer $\left(\mathrm{PdBI}^{\mathrm{g}}\right.$, shown in Figure 1.7) is the previous form of the NOEMA interferometer, operated by the Institut de radioastronomie millimétrique (IRAM). PdBI consisted of $615 \mathrm{~m}$ antennas, located at the Plateu de Bure in the French alps at an elevation of $2550 \mathrm{~m}$. Paper IV uses data from this telescope, from different observing campaigns between 2005 and 2008. Our data cover various molecular lines between 85 and $110 \mathrm{GHz}$, with an angular resolution of $4 "$. 


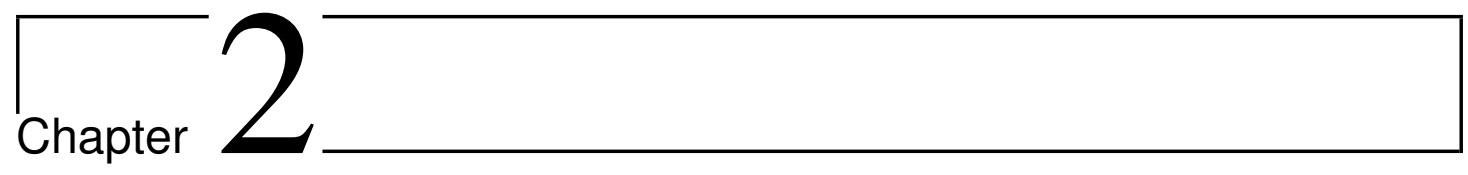

\section{Supernova remnants}

\subsection{Supernovae}

When stars of initial mass $>8 \mathrm{M}_{\odot}$ reach the end of their $\sim 10$-million-year lives they explode as core-collapse ${ }^{\mathrm{a}}$ supernovae (SNe). The core collapses into a neutron star or black hole, while the outer layers of the star are ejected at enormous speeds $\left(\sim 20000 \mathrm{~km} \mathrm{~s}^{-1}\right)$. The explosion releases a large amount of energy: $10^{51}$ ergs, or about as much as the Sun will produce throughout its 10 billion year lifetime. Some of the ejected material (ejecta) undergoes explosive nucleosynthesis, which is the source of many of the heaviest elements found in the Universe, including on Earth. Lighter elements are also present in the ejecta, having formed in the star during its lifetime. The ejecta expands outwards, pushing before it a shock wave which interacts with the circumstellar material expelled by the progenitor star, and then the surrounding ISM. The shock wave will heat the gas it encounters to millions of degrees, ionizing it, and will destroy dust by sputtering (bombarding solid material with fast particles, causing atoms to be ejected from the solid). When this forward shock wave has swept up a mass similar to the ejecta mass, a reverse shock wave is formed, and the object is now called a supernova remnant (SNR). This reverse shock travels back inwards towards the explosion centre at $\sim 2000 \mathrm{~km} \mathrm{~s}^{-1}$, interacting with the ejecta as it goes. When the reverse shock hits the ejecta, the material will be slowed, heated, dissociated, and ionized. The fate of the supernova ejecta is very interesting as it contains large amounts of heavy elements, and understanding the effect of the reverse shock is crucial to determining this fate.

\footnotetext{
${ }^{\text {a } H e n c e f o r t h ~ i n ~ t h i s ~ c h a p t e r ~ I ~ w i l l ~ u s e ~ t h e ~ t e r m ~ s u p e r n o v a ~ t o ~ m e a n ~ s p e c i f i c a l l y ~ c o r e-c o l l a p s e ~ s u p e r n o v a, ~}$ which is caused by the collapse of a massive star, as I will not discuss the other type of supernova (type Ia, caused by a white dwarf star exceeding its Chandrasekhar mass).
} 


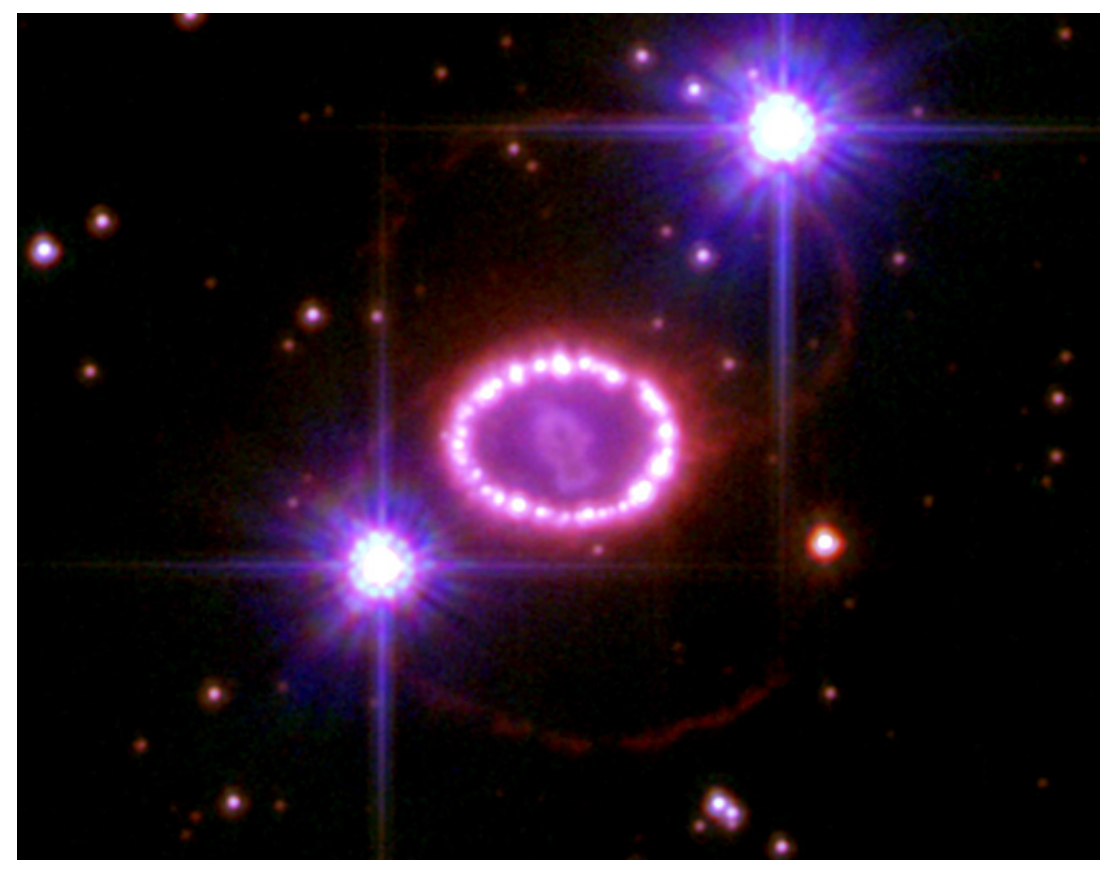

Figure 2.1: Hubble image of SN 1987A, taken in 2007. Image credit: NASA/ESA, P. Challis and R. Kirshner.

\subsubsection{SN 1987A}

February $23^{\text {rd }}, 1987$, was a very special day for the astronomical community. On this day, the light (and neutrinos) from a supernova in the Large Magellanic Cloud, a satellite galaxy to the Milky Way, reached us. It is the nearest supernova that has occurred since we've had the capacity to study them in detail, so SN 1987A (so named because it was the first observed supernova in 1987) was extensively monitored and is still regularly observed today. A Hubble image of this beautiful object is shown in Figure 2.1.

Much of what we know about core-collapse supernovae comes from observations of SN 1987A. Molecules, $\mathrm{CO}$ and $\mathrm{SiO}$, were observed in the SN ejecta some 200 days after the explosion, in vibrational bands, indicating a very high excitation (Danziger et al., 1987; Aitken et al., 1988). However, the molecular emission faded some 600 days later, either because the molecules were destroyed or because they cooled beyond infrared detection. There was also evidence of dust formation in the ejecta from about 500 days post-explosion, seen in the mid-IR (Moseley et al., 1989), with an estimated dust mass of $\sim 10^{-4} \mathrm{M}_{\odot}$. Recent observations with the far-IR Herschel telescope, $\sim 25$ years after the explosion, have revealed large masses of cold dust and molecules: $\sim 0.5 \mathrm{M}_{\odot}$ of dust (Matsuura et al., 2011) and $\sim 0.01 \mathrm{M}_{\odot}$ of CO (Kamenetzky et al., 2013). 


\subsubsection{Molecules and dust in supernova ejecta}

From SN 1987A it is known that dust and molecules are formed soon after the supernova explosion. This is also corroborated by observations of other supernovae (e.g., Kotak et al., 2005, 2006, 2009), so molecule formation is a common occurrence. This is supported by theoretical studies (e.g., Cherchneff \& Dwek, 2009), which show that molecules form quickly and in abundance in SN ejecta. Among these molecules are dust precursors, like $\mathrm{SiO}$, which will bind together into clusters and eventually form dust, a process aided by the cooling of the gas through molecular emission. Hence, molecules are very important for deciding the onset, composition, and mass of SN dust. These dust grains will continue to grow and molecules continue to form as the ejecta cools and expands (Sarangi \& Cherchneff, 2013), as evidenced by the large dust and molecule masses found in SN 1987A decades after the explosion.

The net production of dust in supernovae, taking into account both dust creation in the ejecta and dust destruction by the forward and reverse shocks, is the subject of much debate. The interest in this question stems from the huge dust masses which have been observed in the early Universe (e.g., Bertoldi et al., 2003; Beelen et al., 2006), in galaxies less than a billion years old. This dust had very little time to form, requiring fast dust production processes. In our galaxy, the main dust producers are the evolved AGB stars, which have already lived for some billions of years before moving onto the AGB branch. These stars most likely cannot produce large amounts of dust quickly enough to explain the high$z$ observations (Gall et al., 2011). The stars that explode as supernovae, on the other hand, are more massive and hence have shorter lives, exploding after only $\sim 10$ million years. They are therefore the prime contenders for explaining the large dust masses.

However, this requires that each supernova produce $\sim 0.1-1 \mathrm{M}_{\odot}$ of dust, which is predicted by theoretical models (e.g., Todini \& Ferrara, 2001), but not entirely supported by observations. Observations of warm dust formed some hundreds of days after a supernova find dust masses of only $10^{-5}$ to $10^{-2} \mathrm{M}_{\odot}$ (e.g., Lucy et al., 1989; Sugerman et al., 2006; Szalai \& Vinkó, 2013). But observations of older supernova remnants find cold dust masses of $\sim 0.1 \mathrm{M}_{\odot}$ (Barlow et al., 2010; Matsuura et al., 2011). However, we still do not know the net dust yields of these supernovae, as some supernova dust may be destroyed by the reverse shock, and the forward shock may destroy pre-existing ISM dust. Hopefully, monitoring young SNRs being reprocessed by the reverse shock will provide better constraints on the net dust yield of supernovae.

\subsubsection{Cassiopeia A}

In order to study dust and molecules in SNRs, the SNR needs to be young and near enough that we can distinguish the SN ejecta from the swept up circumstel- 
lar and interstellar material. Cassiopeia A (Cas A) is a prime candidate as it is one of the youngest Galactic supernovae, 330 years old and $3.4 \mathrm{kpc}$ away. Additionally, the reverse shock has only just started to reprocess the ejecta, so there is the opportunity to study the ejecta both pre- and post-shock. As a nearby SNR, Cas A has been extensively studied at a range of wavelengths, from X-ray to radio. The kinematics of the ejecta have been carefully deduced (Milisavljevic \& Fesen, 2013), and a significant mass, $\sim 0.1 \mathrm{M}_{\odot}$, of cool dust has been found (Rho et al., 2008; Barlow et al., 2010). Cas A is also used by theoreticians as a standard SNR to model, and the abundances of various molecules in the SN ejecta have been calculated using chemical kinetic modeling (e.g., Sarangi \& Cherchneff, 2013). Chemical kinetic modeling takes as input the atomic abundances in stratified SN layers from nucleosynthesis modeling, and follows the outcome of hundreds of chemical reactions to determine the abundances of various molecules and clusters as a function of post-explosion time. Such studies have determined that molecules such as $\mathrm{CO}$ and $\mathrm{SiS}$ should be abundant (up to $10 \%$ of the total gas mass) in Cas A, which is an oxygen/silicon-rich SNR.

Fittingly, Cas A is the first supernova remnant where $\mathrm{CO}$ emission has been observed, hundreds of years after the SN explosion. Rho et al. (2009) used the Wide field InfraRed Camera (WIRC) at the Hale Telescope on Mount Palomar to observe the CO 2-0 ro-vibrational (rotational transitions between different vibrational states) band at $2.294 \mu \mathrm{m}$. They detected CO in many small $\left(<1^{\prime \prime}\right)$ knots at the reverse shock at the north and east of the remnant (Figure 2.2). Rho et al. (2012) followed up with spectroscopic observations of the CO fundamental band at $4.65 \mu \mathrm{m}$ with the $A K A R I$ InfraRed Camera (IRC). Using LTE modeling they derive a CO temperature between 1200 and $2400 \mathrm{~K}$, and estimate a total $\mathrm{CO}$ mass of $6 \times 10^{-7} \mathrm{M}_{\odot}$.

The detection of ro-vibrational $\mathrm{CO}$ emission in Cas A paved the way for observations of rotational line emission of $\mathrm{CO}$, and hence the determination of the kinetics, temperature, and density of the ejecta gas, as presented in Paper I.

\subsection{Introduction to Paper I}

In order to probe the physical conditions of the ejecta, several rotational transitions of $\mathrm{CO}$ were observed in a region in the north-west of the supernova remnant Cas A, at the brightest CO knot found by Rho et al. (2009). The previous observations found that the $\mathrm{CO}$ is warm, so we expect high-J rotational transitions to be brighter than low-J transitions. This makes Herschel the ideal telescope to use. In the analysis of these data we use both the rotational diagram method and RADEX-based modeling, as described in $\S 1.2 .3$ and $\S 1.2 .4$.

We find a large column density $\left(\sim 10^{17} \mathrm{~cm}^{-2}\right)$ of warm CO, with two temperature components around 400 and $2000 \mathrm{~K}$. The gas density is constrained to be 


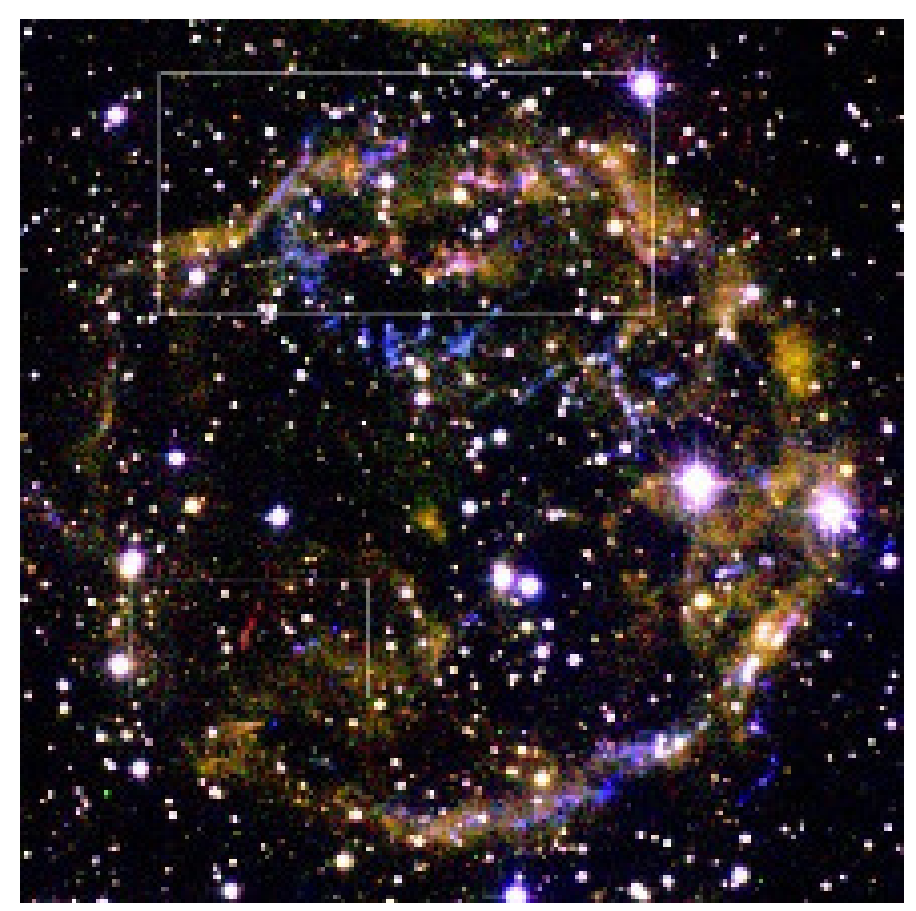

Figure 2.2: Composite near-IR image of Cas A, with ro-vibrational CO emission in red. Areas of excess $\mathrm{CO}$, in the north and east, are marked with boxes. (Image credit: Rho et al., 2009)

high, $\sim 10^{5-6} \mathrm{~cm}^{-3}$, by the RADEX modeling. However, the modelling is limited by the availability of collision rates. In the Cas A knots, the main collision partner for $\mathrm{CO}$ is likely to be oxygen rather than the hydrogen for which we have collision rates.

\subsubsection{Heating mechanisms}

The CO emission appears to originate in a small knot of warm and dense gas. This implies that the knot will be able to cool quickly through the emission of CO lines. As a result, additional heating is required to explain the observed warm temperature. Several heating mechanisms are discussed in Paper I, and are explained below in more detail.

\section{UV heating}

The first heating source considered was the shock front of the reverse shock, where UV photons are emitted from the hot gas $\left(\mathrm{T}>10^{6} \mathrm{~K}\right)$. As the ejecta is oxygen rich, we consider a flux of $F_{o}=n_{o} v_{s}$ ( $n_{o}=$ oxygen density; $v_{s}=$ shock velocity) 
oxygen atoms flowing into the shock. From Borkowski \& Shull (1990) we adopt the model closest to our assumed shock velocity of $200 \mathrm{~km} \mathrm{~s}^{-1}$, which is their model $\mathrm{G}$ with a velocity of $170 \mathrm{~km} \mathrm{~s}^{-1}$. The emergent UV flux they calculate is $29 F_{o}$ photons $\mathrm{cm}^{-2} \mathrm{~s}^{-1}$. These photons have a range of energies, but the vast majority of photons are around $20 \mathrm{eV}$. From this we can calculate a heating flux: $20 \mathrm{eV} \times 29 n_{o} v_{s} \approx 2 \mathrm{erg} \mathrm{cm}^{-2} \mathrm{~s}^{-1}$.

However, photons of energy $20 \mathrm{eV}$ can only penetrate a column density of $\sim 3 \times 10^{17} \mathrm{~cm}^{-2}$. This is similar to the derived column density of $\mathrm{CO}$, so the total gas column density must be much larger. Hence, UV photons cannot penetrate and heat the full gas column.

\section{X-ray heating}

Both the forward and reverse shock, when traveling through tenuous gas, create a hot plasma that will slowly cool through X-rays. The average observed X-ray luminosity over the remnant is $5.5 \times 10^{37} \mathrm{erg} \mathrm{s}^{-1}$ (Hartmann et al., 1997). Dividing this by the size of the remnant $\left(\sim 4^{\prime}\right.$ at $\left.3.4 \mathrm{kpc}\right)$, we find an X-ray heating flux of $0.2 \mathrm{erg} \mathrm{cm}^{-2} \mathrm{~s}^{-1}$. These photons have a typical energy of $\sim 2 \mathrm{keV}$, meaning they can heat a larger column density $\left(\sim 3 \times 10^{19} \mathrm{~cm}^{-2}\right)$ than the UV photons.

\section{Electron conduction}

The hot shocked plasma could also heat the dense knot via heat conduction. The classical expression for heat conduction by electrons is

$$
Q=K(T) \frac{d T}{d r}
$$

where $K(T)$ is roughly constant and equal to $\sim 6 \times 10^{-7} T^{5 / 2} \mathrm{erg} \mathrm{s}^{-1} \mathrm{~K}^{-7 / 2} \mathrm{~cm}^{-1}$ (Tielens, 2005, p. 448-449). Note that this classical expression requires that the mean free path of the electrons is small compared with the length scale of the temperature gradient. For the parameters we adopt, this is indeed the case with a mean free path of $\sim 10^{12} \mathrm{~cm}$ and a length scale of $5 \times 10^{13} \mathrm{~cm}$.

Setting the temperature gradient equal to $T / \delta R$ with the temperature $T=10^{7} \mathrm{~K}$ and the length scale $\delta R$ given by the gas column density divided by the gas density, $N / n=5 \times 10^{13} \mathrm{~cm}$, we find an energy flux of $\sim 4 \times 10^{4} \mathrm{erg} \mathrm{cm}^{-2} \mathrm{~s}^{-1}$. This exceeds the UV and X-ray heating flux by several orders of magnitude, and is thus most likely the main heating mechanism.

Heat conduction is, however, extremely temperature sensitive. If the temperature of the hot plasma were decreased by an order of magnitude, to $10^{6} \mathrm{~K}$, the energy flux would be only $\sim 10 \mathrm{erg} \mathrm{cm}^{-2} \mathrm{~s}^{-1}$. This still surpasses the other heating mechanisms, but not by nearly as much. 
The heat conduction energy into the knot will be balanced by mass evaporation from the surface of the knot. The mass-loss rate of the knot will be

$$
\dot{M}_{k}=\frac{16 \pi}{25} \frac{\mu}{k_{B}} K(T) T_{h}^{5 / 2} R_{k}
$$

where $\mu$ is the average mass of the gas (taken to be $16 \mathrm{Da}^{\mathrm{b}}$, for an oxygen gas), $T_{h}$ is the temperature of the hot electrons (here taken to be $10^{7} \mathrm{~K}$ ), and $R_{k}$ the size of the knot. For our CO knot we find $\dot{M}_{k} \sim 6 \times 10^{-5} \mathrm{M}_{\odot} \mathrm{yr}^{-1}$, corresponding to an evaporation time of $\sim 2000$ years. The shock will take only $\sim 50$ years to cross the knot, so the knot will not evaporate significantly during the shock interaction.

\subsection{Future prospects}

To continue the work on the reverse shock conditions of Cas A, observations are underway with the FIFI-LS instrument aboard the SOFIA airborne observatory of oxygen fine structure lines - [O I] at $63 \mu \mathrm{m}$ and [O III] at 52 and $88 \mu \mathrm{m}$ - at three CO-bright points in the remnant. With these observations we aim to understand the characteristics and interrelationship of the molecular and neutral/ionic atomic gas. The data will shed light on the questions of how ejecta knots are processed by the reverse shock and whether such knots can protect supernova dust, allowing it to survive into the ISM.

An extension of the work on Cas A is to search for molecules in other supernova remnants. SN 1987A is an obvious candidate, as $\mathrm{CO}$ and $\mathrm{SiO}$ have already been discovered in its ejecta (Kamenetzky et al., 2013). I am co-lead of an accepted ALMA proposal to study the molecules $\mathrm{SiS}, \mathrm{SO}$ and $\mathrm{SiO}$ (and their isotopologues) in the ejecta of SN 1987A. These molecules are expected to form in abundance in supernova ejecta, from modelling by Sarangi \& Cherchneff (2015). The observations cover several transitions of each molecule, and so will allow for the determination of the physical conditions and kinematics of the gas, as well as various isotopic ratios. Hence, we will be able to test predictions of ejecta chemistry, clumpiness, and nucleosynthesis. There is also a plan to follow up and extend these observations in future ALMA cycles: more transitions will provide further constraints on ejecta conditions and molecular formation zones, and a wider range of molecules will further constrain the chemistry and nucleosynthesis.

\footnotetext{
${ }^{\mathrm{b}}$ Dalton or unified atomic mass unit, defined as one twelfth of the mass of a neutral carbon-12 atom.
} 


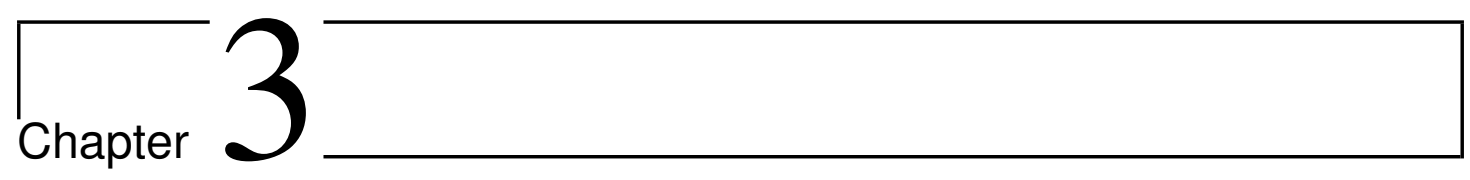

\section{Massive evolved stars}

When massive stars near the end of their lives their stellar winds become very vigorous, ejecting up to $10^{-3} \mathrm{M}_{\odot}$ of material per year. Despite their rarity, such stars have a huge impact on the ISM. Molecules can be used as tracers of this wind, probing the mass-loss rate and the kinematics of the gas. A commonly observed molecule is $\mathrm{CO}$, due to its abundance and excellent observational properties, as discussed in $\S 1.2$. The distribution of this circumstellar gas will affect the future evolution and (very likely) explosion of a massive star as a supernova, so studying these late evolutionary stages is vital to understanding the lives and deaths of massive stars.

\subsection{Yellow Hypergiants}

General references: Oudmaijer et al. (2009); de Jager (1998)

When stars of mass $>8 \mathrm{M}_{\odot}$ run out of hydrogen in their cores at an age of $\sim 10^{7}$ years, they move off the main sequence to become Red Supergiant (RSG) stars. This stage, and later evolutionary stages, are characterized by intense mass-loss. This mass-loss shapes the circumstellar envelope, which will impact the geometry of an eventual supernova explosion. The amount of mass lost determines whether the star explodes as a supernova at all, because if it loses too much mass the core will not be heavy enough to undergo catastrophic collapse.

After the RSG branch, a massive star can evolve in several ways depending on its composition and other characteristics. Some stars make "excursions" around the HR diagram, eventually returning to the RSG branch. Others evolve into Luminous Blue Variable (LBV) and then Wolf Rayet (WR) stars, which are very hot stars losing a lot of mass via fast stellar winds. Along the evolutionary progression from RSG to WR stars, which is expected to take several hundred 


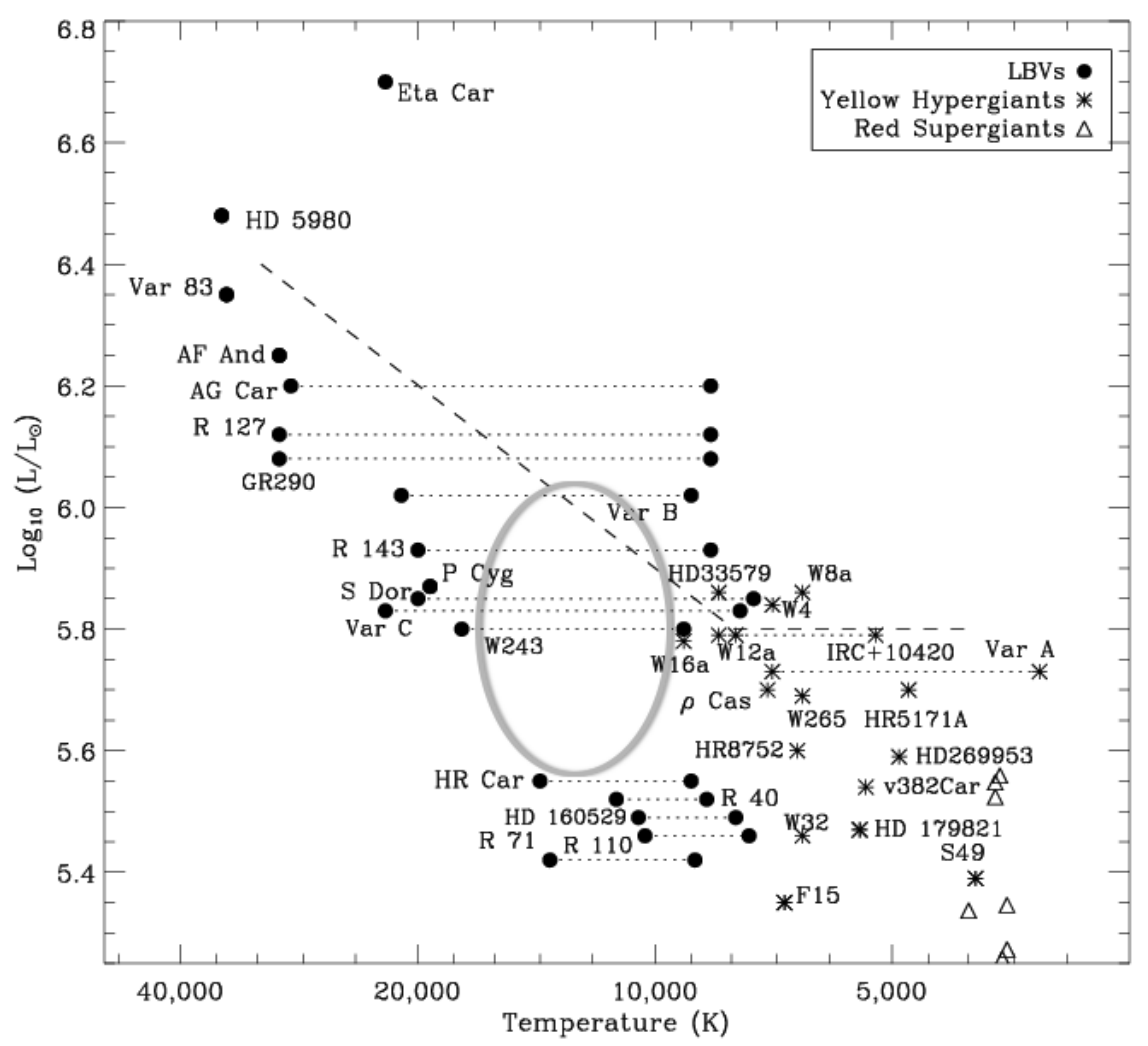

Figure 3.1: The high-luminosity part of the HR diagram, where the Yellow Hypergiants are found. The approximate location of the Yellow Void is indicated with a grey ellipse. (Image credit: Oudmaijer et al., 2009)

to a few thousand years (Garcia-Segura et al., 1996), there is the Yellow Hypergiant (YHG) stage. The number of known Yellow Hypergiant stars is small, partly because of the short duration of the YHG stage, and partly because they are difficult to identify. One defining characteristic of a YHG star is an absolute magnitude, $M_{V}$, brighter than -7, and the determination of $M_{V}$ requires an accurate distance measurement. Another feature of YHGs is broad $\mathrm{H} \alpha$ emission components, which implies large-scale photospheric motion and an extended envelope. This, in turn, implies a large mass-loss rate. So YHGs are intrinsically bright, with typical luminosities around $10^{6} \mathrm{~L} \odot$, and are losing a lot of mass. They have temperatures between $\sim 7000-10000 \mathrm{~K}$, so on the HR diagram they lie between RSGs and LBV/WR stars (Figure 3.1).

There is a gap in the HR diagram, an area with very few stars just bluewards of the YHGs, called the Yellow Void. The Yellow Void is believed to be associated with inherently unstable stellar parameters, so that YHGs evolving 
towards it will not be able to enter the Void, and will instead "bounce" back redwards. This "bouncing" against the Yellow Void is another unique characteristic of the Yellow Hypergiants. "Bounces" have been observed to be associated with periods of increased mass-loss, which are believed to cause the subsequent redward motion: the wind becomes optically thick, essentially forming a cool pseudo-photosphere around the star. After the wind clears, the star can once again move towards the Yellow Void, until its photosphere becomes unstable and it enters another period of increased mass-loss. The star HR 8752, for example, has undergone at least two "bounces" over a period of 30 years (de Jager \& Nieuwenhuijzen, 1997).

The photospheric instability of the Yellow Void is associated with the effective photospheric acceleration, $g_{\text {eff }}$. When $g_{\text {eff }}$ is close to zero, as is the case at the Yellow Void, the atmosphere is more or less detached from the star's gravitational attraction. This means the star will pulsate with supersonic velocity amplitudes, allowing for ejection of gas at supersonic speeds and greatly enhanced mass-loss during that part of the pulsation cycle. The pulsation period is typically $\sim 500$ days, and the star can eject huge amounts of gas on a timescale of about a decade. It is thought that once a YHG has ejected enough mass its stellar envelope will be able to survive the instabilities of the Yellow Void, and the star will evolve to become an LBV on the other side of the Void. Hence the YHG stage links the comparatively well understood RSG and LBV/WR phases, both evolutionarily and with regard to the formation of circumstellar structures. It is important to study YHGs for this reason, despite the short duration of the phase, but the varied properties of YHG stars poses a challenge.

The variation in the properties of YHGs is caused by the movement of the stars in the HR diagram, and their "bouncing" against the Yellow Void, but they have a few features in common: large luminosities, and large mass-loss rates. This, combined with their past RSG phase, means YHGs should have significant circumstellar envelopes. But very few have been found to have extended shells: the famous $\rho$ Cas, for example, shows no extended emission. One of the most studied YHGs, IRC +10420 , is one of the few that shows extended circumstellar shells. It is also significant in its large outflow velocity $\left(\sim 40 \mathrm{~km} \mathrm{~s}^{-1}\right)$ and huge mass-loss rate $\left(>10^{-4} \mathrm{M}_{\odot} \mathrm{yr}^{-1}\right)$. On the largest scales, IRC+10420 has largely spherically symmetric circumstellar shells, but closer to the star the more recently ejected features show lots of structure (Castro-Carrizo et al., 2007). These structures appear to have been ejected by short bursts of mass-loss in random directions, perhaps indicative of explosive "bounces" against the Void. Another of the few YHGs with extended circumstellar shells is the newly reclassified IRAS 17163-3907 (IRAS 17163), which is the subject of Papers II and III. 


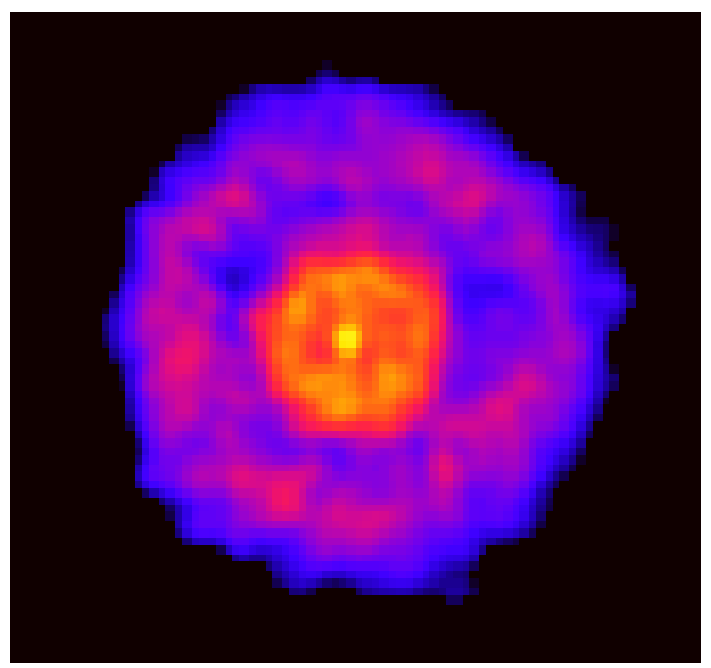

Figure 3.2: 8.6 $\mu \mathrm{m}$ VISIR image of IRAS 17163-3907. Image credit: Lagadec et al. (2011).

\subsubsection{IRAS 17163-3907}

IRAS 17163 was only recently identified as a Yellow Hypergiant star, despite being one of the brightest infrared sources in the sky, because its distance was not well constrained. The star was discovered by Henize (1976), and classified as a post-AGB star by Le Bertre et al. (1989). They assumed a distance less than $1 \mathrm{kpc}$ based on a positive radial velocity, inconsistent with Galactic rotation, which means the star is most likely in the solar neighborhood. The close distance was backed up by the fact that the star was resolved by IR speckle interferometry.

However, a new distance measurement was made by Lagadec et al. (2011) based on interstellar absorption lines in the optical spectrum of IRAS 17163. The absorption line velocity suggests the absorbing gas is $3.6 \mathrm{kpc}$ away, if it follows Galactic rotation, giving a lower limit on the distance to the star. An upper limit on the distance was also derived, based on the maximum visual extinction, to be $4.7 \mathrm{kpc}$. The new distance of $\sim 4 \mathrm{kpc}$ also increases the derived luminosity of the star to $5 \times 10^{5} \mathrm{~L}_{\odot}$, placing IRAS 17163 among the Yellow Hypergiants on the HR diagram.

The YHG status of IRAS 17163 is further backed up by dust measurements: Lagadec et al. (2011) found two concentric spherical dust shells within 5" of the star using mid-IR observations, with a huge warm $(\sim 200 \mathrm{~K})$ dust mass of 0.04 $\mathrm{M}_{\odot}$, inspiring the nickname the Fried Egg nebula. These dust shells can be seen in Figure 3.2. Hutsemékers et al. (2013) used Herschel to look in the far-IR and discovered a 50 " dust ring around the star containing $\sim 0.17 \mathrm{M}_{\odot}$ of $\operatorname{cool}(\sim 60 \mathrm{~K})$ dust. This implies a total ejected mass of $\sim 10 \mathrm{M}_{\odot}$, depending on the assumed gas-to-dust mass ratio (here: 50 ), over the past $\sim 10000$ years. The 50 " ring was 
most likely ejected during the star's previous RSG phase, but the warm dust close to the star is estimated to have been ejected in several mass-loss episodes within the past $\sim 400$ years, consistent with the YHG phase. Paper II presents an optical spectrum of IRAS 17163, providing further evidence it is indeed a Yellow Hypergiant, along with APEX CO observations of its circumstellar material. Paper III probes the CO emission in more detail with ALMA ACA observations.

\subsection{Introduction to Paper II}

In order to probe the kinematics of the circumstellar envelope and constrain the mass-loss rate, IRAS 17163 was observed in CO 2-1 and 3-2 using the APEX telescope. A complex CO line shape is found at a radial velocity around $+65 \mathrm{~km} \mathrm{~s}^{-1}$, consisting of two peaks and a broad $\left(\sim 50 \mathrm{~km} \mathrm{~s}^{-1}\right)$ underlying component.

An optical spectrum from the Mercator telescope is also presented, and compared with the optical spectrum of the YHG IRC+10420. The similarity between the two spectra further supports the classification of IRAS 17163 as a Yellow Hypergiant. The optical emission lines have radial velocities around $+18 \mathrm{~km} \mathrm{~s}^{-1}$, significantly offset from the velocity of the $\mathrm{CO}$ emission. Proposed explanations of the velocity discrepancy include a binary companion and pulsation of the stellar atmosphere.

\subsection{Introduction to Paper III}

ALMA ACA observations of CO 2-1 were obtained to shed light on the complex circumstellar structure of IRAS 17163. They reveal a faint broad plateau at the star, centered on $+18 \mathrm{~km} \mathrm{~s}^{-1}$ with a total width of $\sim 250 \mathrm{~km} \mathrm{~s}^{-1}$, resolving the $\mathrm{CO} /$ optical velocity discrepancy from Paper II and finally confirming the systemic velocity of IRAS 17163 to be $+18 \mathrm{~km} \mathrm{~s}^{-1}$. We model this plateau as isotropic mass-loss from the star, as described in $\S 3.3 .1$ below, to find an expansion velocity of $100 \pm 10 \mathrm{~km} \mathrm{~s}^{-1}$ and a mass-loss rate of $1.2 \pm 0.3 \times 10^{-4} \mathrm{M}_{\odot} \mathrm{yr}^{-1}$.

The observations also reveal a clumpy CO ring, matching the 50" Herschel dust ring (Hutsemékers et al., 2013). However, the kinematics of this ring are not consistent with an expanding shell. The velocity structure is more like that of a torus, at a shallow inclination, like the famous ring around SN 1987A. Such structures have been detected around a few evolved stars, and may require a binary companion to form.

The bright emission around $+60 \mathrm{~km} \mathrm{~s}^{-1}$, seen with APEX in Paper II, is a spur extending from the star to the clumpy $\mathrm{CO}$ ring. There appears to be no counterpart on the other side of the star, though the ISM contamination around $-20 \mathrm{~km} \mathrm{~s}^{-1}$ makes it difficult to confirm. We obtained an APEX spectrum of

${ }^{13} \mathrm{CO}$ in order to calculate the ${ }^{12} \mathrm{C} /{ }^{13} \mathrm{C}$ ratio and elucidate the origin of the spur 
material: a ratio around 10 would suggest a red supergiant origin, while the ISM around IRAS 17163 has a ratio of $\sim 40$. We find a ${ }^{12} \mathrm{CO} /{ }^{13} \mathrm{CO}$ ratio of $13_{-5}^{+10}$, indicating the spur does indeed originate from the star. How such a massive unipolar ejection, over several thousand years, could have occurred remains a mystery. It is also at odds with the circular symmetry of the dust emission, at both smaller and larger scales.

\subsubsection{Modeling}

In order to estimate the mass-loss rate of IRAS 17163, we use a simple model of an expanding circumstellar envelope where the radiative transfer is solved using a Monte Carlo method (Bernes, 1979; Schöier \& Olofsson, 2001). This model assumes that the envelope expands at a constant velocity and is formed by a constant, isotropic, and smooth mass-loss. It incorporates rotational energy levels up to $\mathrm{J}=40$ for the vibrational ground state and the first vibrationally excited state, and uses collisional cross-sections from Yang et al. (2010). In our modeling we take the stellar parameters (distance, $\mathrm{T}_{\text {eff }}$, luminosity) from Lagadec et al. (2011), and assume a $[\mathrm{CO}] /\left[\mathrm{H}_{2}\right]$ ratio of $10^{-4}$, a turbulent velocity of $1 \mathrm{~km} \mathrm{~s}^{-1}$, and a gas-to-dust ratio of 40 (cf. Hutsemékers et al., 2013). Note that the gas-todust ratio is only used in the energy balance calculation.

We fit the mass-loss rate and expansion velocity by executing models over a parameter grid where the mass-loss rate is varied between $9 \times 10^{-5}$ and $2 \times 10^{-4}$ $\mathrm{M}_{\odot} \mathrm{yr}^{-1}$, and the expansion velocity between 85 and $125 \mathrm{~km} \mathrm{~s}^{-1}$. We then determined the best fit model by $\chi^{2}$ minimisation to the parts of the spectrum not contaminated with ISM or spur emission, to find an expansion velocity of $100 \pm 10 \mathrm{~km} \mathrm{~s}^{-1}$ and a mass-loss rate of $1.2 \pm 0.3 \times 10^{-4} \mathrm{M}_{\odot} \mathrm{yr}^{-1}$. The errors are the statistical errors corresponding to the $1 \sigma \chi^{2}$ error ellipse, i.e., where the parameters correspond to a model with a $\chi^{2}$ value equal to $\chi_{\min }^{2}+1$.

The mass-loss rate estimate has further uncertainty associated with the model parameters, such as the distance to the star and the assumption of constant and isotropic mass-loss, which are not taken into account. Note also that the model was developed for the circumstellar envelopes of AGB stars, and hence may not be strictly applicable to our YHG star. For example, it assumes an entirely molecular gas envelope, whereas the envelope of IRAS 17163 might be significantly atomic due to the high stellar temperature. On the other hand, this method provides an order of magnitude estimate of the mass-loss rate, allowing us to make comparisons to other massive evolved stars. Furthermore, previous modelling of the YHG IRC +10420 which finds similar mass-loss rates (CastroCarrizo et al., 2007; Dinh-V.-Trung et al., 2009) also used models developed for AGB envelopes. 


\subsection{Future prospects}

The ALMA observing programme which yielded the ACA data for Paper III has now been completed, with the full array data having a spatial resolution of $1.5^{\prime \prime}$. The combination of the full array data with the ACA and total power observations will give a more complete picture of the surroundings of IRAS 17163, and allow us to study its peculiar features in greater detail. There are also several other YHGs with detected circumstellar material which could be similarly studied with ALMA.

Further optical and near-infrared observations of IRAS 17163 with the SPHERE instrument at the VLT are also forthcoming. With more detailed observations of the dust shells within $2.5^{\prime \prime}$ of the star, coupled with the high resolution ALMA data, we can directly compare the gas and dust and model the recent behavior of this star. There are also plans to monitor the optical spectrum of IRAS 17163 to look for short term variability and potential evidence of a binary companion. 


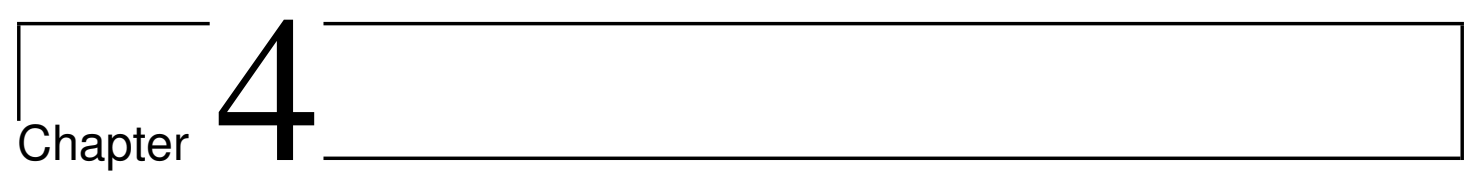

\section{Galactic chemical evolution}

When stars lose mass, through stellar wind or explosion, their nucleosynthesis products get incorporated into the ISM. This is the source of the chemical enrichment of a galaxy: over time, it gains more and more metals. Different stars will enrich the ISM with different elements, and on different timescales. This is mainly due to the stellar mass, as the more massive a star is, the faster it will evolve and the larger the range of elements it can produce. Young galaxies will mainly show enrichment due to massive stars, as the lighter stars will not yet have had time to evolve off the main sequence and affect the ISM. Hence, by studying galaxies of different ages, we can constrain the effects from different sources of chemical enrichment.

In order to study this chemical enrichment, one could measure the abundances of various elements in galaxies at different ages of the Universe. However, determining the overall abundance of an element can be difficult, as different elements will be preferentially found in different states, e.g., incorporated in molecules, as neutral atoms, or in various states of ionisation. Luckily, different sources of chemical enrichment don't just produce different abundances of elements, but also different abundances of isotopes of those elements. Determining isotopic ratios from atomic lines in optical spectra requires very high spectral resolution, as the lines typically lie very close together. On the other hand, when isotopes are incorporated into molecules they can significantly change the frequency of their transitions and are therefore easily distinguishable.

When molecules are formed fractionation can occur, which is when molecules are preferentially formed with a specific isotope of a given element. Fractionation can also be due to selective isotopic photodissociation. It occurs mainly with light elements, for which the addition of a neutron causes a large relative change in the atomic mass and hence a corresponding change in the bonding energy of the molecule. Hydrogen/deuterium fractionation is common, and in cold, dense clouds carbon and nitrogen fractionation is also seen (Roueff et al., 
2015, and references therein). However, heavy elements or those in warm environments are generally not affected by fractionation, so the relative isotopologue abundances of a molecule will be proportional to the isotopic ratio of that element. Determining the isotopic ratios of various elements at different ages of the Universe puts constraints on the physics of stellar evolution and the rates of star formation and destruction.

\subsection{Chemical evolution models}

Theoretical models of galactic chemical evolution have been developed to explain the elemental abundances and isotopic ratios measured in different galaxies. These take into account the contributions from different types of stars over different timescales. Often these models will use metallicity as a proxy for time, as this is easier to relate to observational results. Metallicity is generally defined as the iron content relative to hydrogen, $[\mathrm{Fe} / \mathrm{H}]$, as iron is an abundant heavy element and often the easiest to measure. The abundance ratio is expressed as the logarithm of the iron abundance in the gas compared with that of the Sun:

$$
[\mathrm{Fe} / \mathrm{H}]=\log _{10}\left(\frac{N_{F e}}{N_{H}}\right)_{g a s}-\log _{10}\left(\frac{N_{F e}}{N_{H}}\right)_{\text {Sun }}
$$

where $N_{F e}$ is the number of iron atoms per volume and $N_{H}$ the number of hydrogen atoms per volume. Hence a positive value of $[\mathrm{Fe} / \mathrm{H}]$ corresponds to a higher metallicity than that of the Sun, and a negative value to a lower than Solar metallicity.

Kobayashi et al. (2011) produce one of the few chemical evolution models to predict the evolution of different isotopes for a wide range of elements. They calculate the evolution of the gas fraction and metallicity of the Milky Way as a function of time, which depends on the star formation rate as well as infall and outflow of matter. The star formation rate (SFR) is taken to be proportional to the gas fraction, and they adopt the initial mass function (IMF) from Kroupa (2008) of $n_{m} \propto m^{-x}$, where $n_{m}$ is the number of stars of mass $m$, and the slope $x$ is given for three separate mass ranges: $x=2.3$ for $0.5 \mathrm{M}_{\odot} \leqslant m \leqslant 50 \mathrm{M}_{\odot}, x=1.3$ for $0.08 \mathrm{M}_{\odot} \leqslant m \leqslant 0.5 \mathrm{M}_{\odot}$, and $x=0.3$ for $0.01 \mathrm{M}_{\odot} \leqslant m \leqslant 0.08 \mathrm{M}_{\odot}$. They also include different star formation histories for different parts of the Milky Way, in order to recreate the observed differences in their metallicity distribution function (MDF) ${ }^{\mathrm{a}}$. For example, in the solar neighborhood star formation takes place over 13 Gyr (billion years) with the SFR peaking at $[\mathrm{Fe} / \mathrm{H}] \sim-0.2$. There is also some infall of primordial (zero metallicity) gas from outside the Galactic disk. In contrast, the bulge has a shorter star-formation timescale, only $3 \mathrm{Gyr}$, before it is truncated by a galactic wind.

\footnotetext{
${ }^{\mathrm{a}}$ An MDF gives the proportion of stars at each metallicity
} 
The models of Kobayashi et al. sum all the contributions of different stars, which depend on their initial masses. Stars with initial masses between 0.8 and $8 \mathrm{M}_{\odot}$ become asymptotic giant branch (AGB) stars, which produce a large fraction of light elements such as $\mathrm{C}, \mathrm{N}$, and F. They also produce a significant amount of s-process elements (see $\S 1.1 .2$ ), and expel their nucleosynthesis products into the ISM via stellar winds. Some elements, such as ${ }^{15} \mathrm{~N}$, are destroyed rather than created in AGB stars. In cases where the element yield would be negative, it is instead set to zero to avoid numerical problems, which may affect the derived isotopic ratios, e.g., the ratio ${ }^{14} \mathrm{~N} /{ }^{15} \mathrm{~N}$.

After expelling most of their material, AGB stars leave behind a white dwarf star. If a white dwarf exceeds the Chandrasekhar mass of $\sim 1.4 \mathrm{M}_{\odot}$, either by accreting material (single degenerate scenario) or merging with another white dwarf (double degenerate scenario), it will explode as a Type Ia supernova. The lifetimes of double degenerate systems are too short to reproduce the chemical evolution of the solar neighborhood, as they produce too much Fe at early times. Hence, Kobayashi et al. consider only a single degenerate scenario. Type Ia supernovae produce large amounts of elements such as $\mathrm{Si}, \mathrm{S}$, and Fe.

Stars with slightly larger initial masses, between 8 and $10 \mathrm{M}_{\odot}$, become super AGB stars, for which metal production is predicted to be very small. Hence, Kobayashi et al. assume these stars produce no metals, but they do leave behind a white dwarf star which could potentially explode as a Type Ia supernova. Stars with initial masses between 10 and $50 \mathrm{M}_{\odot}$ will expel material as massive evolved stars before ending their lives as core-collapse supernovae. These produce large amounts of $\mathrm{O}$, for example, and all r-process elements. Stars with initial masses between $\sim 100$ and $300 \mathrm{M}_{\odot}$ end their lives as pair-instability supernovae, which are predicted to produce large amounts of, e.g., $\mathrm{S}$ and Fe. However, as the number of such stars is expected to be very small, Kobayashi et al. do not include their contribution in the models.

\subsection{Molecular absorption at high redshift}

Isotopic ratios provide valuable probes of the enrichment history of the Universe, and high redshift sources provide some of the most valuable data. When studying the gas in distant galaxies, an absorbing galaxy in front of a quasar is the ideal source. As the line strength is not affected by distance (see $\S 1.2 .2$ ) even faint galaxies can be studied in high detail. However, the need for chance alignment with a background quasar means there are few such systems. Two of the most well studied are presented below. 


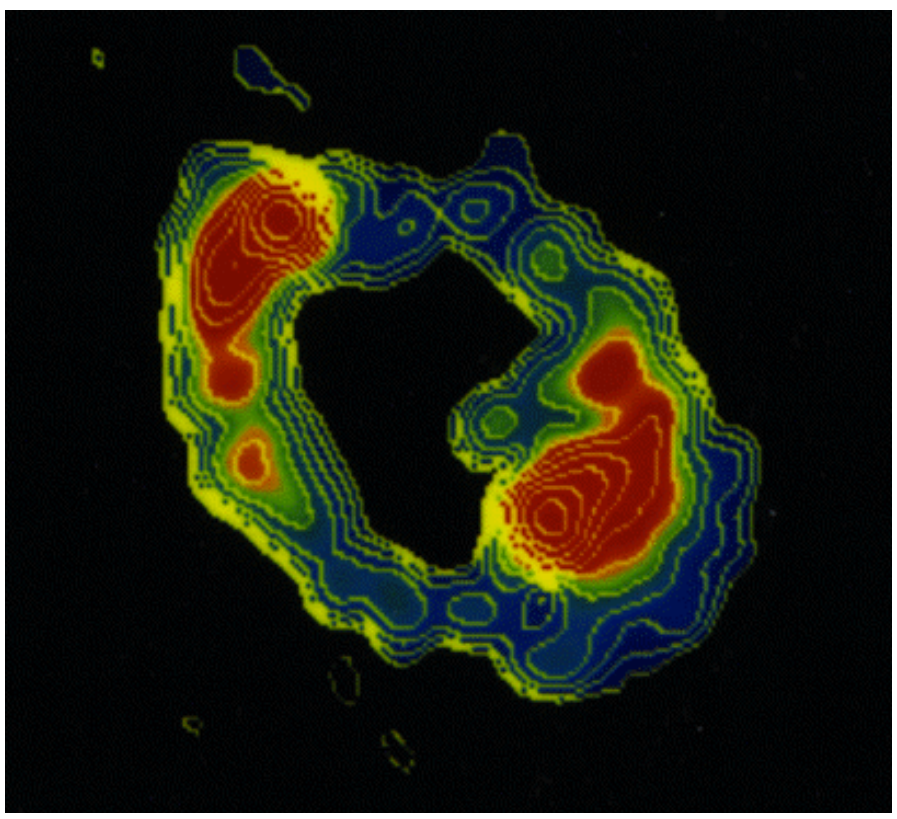

Figure 4.1: MERLIN $5 \mathrm{GHz}$ image of the PKS 1830-211 Einstein ring. North is up and East is left in the image. Image credit: Australia Telescope National Facility, CSIRO.

\subsubsection{The $\mathrm{z}=0.89$ absorber towards PKS 1830-211}

PKS 1830-211 is a blazar at $\mathrm{z}=2.5$ being lensed by a nearly face-on spiral galaxy at $\mathrm{z}=0.89$ (hereafter referred to as MA0.89), discovered by Wiklind \& Combes (1996). MA0.89 acts as a gravitational lens, splitting the image of the quasar into two main components, NE and SW, embedded in an Einstein ring, as shown in Figure 4.1. The two main images are separated by about $1^{\prime \prime}$, and intercept the absorbing galaxy at about 4 and $2 \mathrm{kpc}$ from the galactic centre for the NE and SW images, respectively. The SW image shows absorption lines from $>50$ molecular species (Menten et al., 1999; Muller et al., 2006, 2011, 2014; Müller et al., 2015), and some of their isotopologues. These molecules show rotational temperatures consistent with the $\mathrm{CMB}$ temperature at $\mathrm{z}=0.89,5.14 \mathrm{~K}$, but the kinetic temperature is much warmer, $\sim 80 \mathrm{~K}$. Hence the observed transitions are subthermally excited, which is consistent with a modest gas density of $\sim 10^{3} \mathrm{~cm}^{-3}$. The absorbing gas is similar to diffuse or translucent clouds in the Milky Way. Towards the NE image the molecular column density is about one order of magnitude lower than towards the SW, so only a few molecules are detected there (Muller et al., 2011).

The isotopic ratios measured in MA0.89 are quite different from those found in the local ISM. Muller et al. (2006) found low values of the ${ }^{12} \mathrm{C} /{ }^{13} \mathrm{C},{ }^{14} \mathrm{~N} /{ }^{15} \mathrm{~N}$, ${ }^{16} \mathrm{O} /{ }^{18} \mathrm{O}$, and ${ }^{32} \mathrm{~S} /{ }^{34} \mathrm{~S}$ ratios, at least a factor of two lower than in the local ISM. 


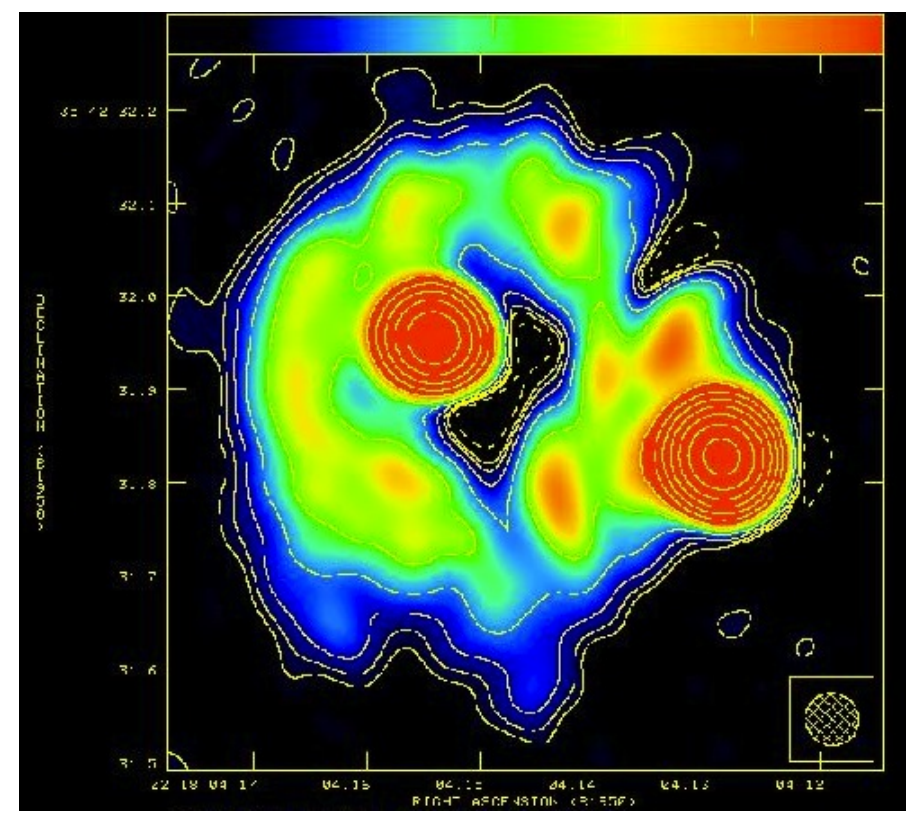

Figure 4.2: MERLIN $5 \mathrm{GHz}$ image of B0218+357. North is up and East is left in the image. Image credit: Biggs et al. (2001).

They also find a ${ }^{18} \mathrm{O} /{ }^{17} \mathrm{O}$ ratio a factor of 2-3 larger than in the local ISM. All these results are consistent with a young galaxy, mainly enriched by massive stars.

\subsubsection{The $\mathrm{z}=0.68$ absorber towards $\mathrm{B} 0218+357$}

$\mathrm{B} 0218+357$ is a blazar at $\mathrm{z}=0.94$ which is being lensed by a molecular absorber at $\mathrm{z}=0.68$, hereafter called MA0.68. This produces two images (A and B) separated by $0.33^{\prime \prime}$, and an Einstein ring (Patnaik et al., 1993), as shown in Figure 4.2. The brightest image, A, in the West, is well away from the ring, while image $\mathrm{B}$ is close to the centre of the ring. Molecular absorption from species such as $\mathrm{CO}, \mathrm{HCO}^{+}, \mathrm{HCN}, \mathrm{H}_{2} \mathrm{O}, \mathrm{NH}_{3}$, and $\mathrm{H}_{2} \mathrm{CO}$ has been found toward image A only (Wiklind \& Combes, 1995; Muller et al., 2007). MA0.68 is a nearly face-on spiral, and the absorption occurs about $2 \mathrm{kpc}$ from the galaxy's center.

Observations of ammonia have shown that the absorbing gas is warm and diffuse (Henkel et al., 2005), similar to MA0.89. A redshift of $\mathrm{z}=0.68$ corresponds to a look-back time of nearly half the age of the Universe, so this galaxy has had much less time for chemical enrichment and evolution than, e.g., the Milky Way. Hence its ISM should show enrichment mainly by massive stars. MA0.68 is the subject of Paper IV. 


\subsection{Introduction to Paper IV}

We have obtained PdBI observations of a galaxy at $\mathrm{z}=0.68$, located in front of the blazar B 0218+357, and detect absorption lines from $\mathrm{HCO}^{+}, \mathrm{HCN}, \mathrm{CS}, \mathrm{H}_{2} \mathrm{~S}$, and some of their isotopologues. We use these to measure isotopic ratios of ${ }^{32} \mathrm{~S} /{ }^{34} \mathrm{~S}$ and ${ }^{18} \mathrm{O} /{ }^{17} \mathrm{O}$, as well as the double ratios ${ }^{13} \mathrm{C} /{ }^{18} \mathrm{O}$ and ${ }^{13} \mathrm{C} /{ }^{15} \mathrm{~N}$ where the main isotopologue is saturated. We also estimate the ${ }^{12} \mathrm{C} /{ }^{13} \mathrm{C}$ ratio, and hence ${ }^{14} \mathrm{~N} /{ }^{15} \mathrm{~N}$ and ${ }^{16} \mathrm{O} /{ }^{18} \mathrm{O}$. We find all these ratios to be similar to those found in the PKS 1830-211 absorber at $\mathrm{z}=0.89$. They differ significantly from isotopic ratios in the solar neighborhood, but are similar to several starburst galaxies, which is consistent with enrichment mainly by massive stars.

\subsubsection{Determining isotopic ratios}

For optically thin lines, absorption intensities $\left(\mathrm{I}_{v}\right)$, measured from the continuum level, can be converted to optical depths $\left(\tau_{v}\right)$ according to:

$$
\tau_{v}=-\ln \left(1-\frac{I_{v}}{f_{c} I_{b g}}\right)
$$

where $I_{b g}$ is the intensity of the background continuum source, and $f_{c}$ the source covering factor. As the observations cannot resolve images $\mathrm{A}$ and $\mathrm{B}$ of the quasar and absorption only occurs in front of image $A$, we assume a flux ratio $F_{A} / F_{B}=4.2$ and hence a continuum intensity for image $\mathrm{A}$ of 0.81 , and a source covering factor of 0.8 (see Paper IV for details).

We convert the absorption lines to optical depths, and fit each line profile with the same gaussian components, scaled by a multiplicative factor. Hence we assume the same excitation, continuum illumination, fractional abundances and isotopic ratios for all velocity components. Next we calculate an integrated opacity for each line, and convert them to column densities. We derive isotopic ratios using a Monte Carlo routine to divide the column densities, including uncertainties, of relevant pairs of lines. This method does not require the assumption of symmetric errors, giving more realistic uncertainties for each isotopic ratio.

\subsection{Future prospects}

Further studies of the isotopic ratios towards B 0218 will be best achieved with detailed ALMA observations, and the resubmission of a proposal to do this is planned. The resolution and sensitivity of ALMA would allow us to observe many more molecules and their isotopologues, and multiple transitions of the same molecule in order to derive physical conditions. There are also a few 
other galaxies seen in absorption towards quasars, for example the absorber at $\mathrm{z}=0.25$ towards PKS 1413 +135 , and there are searches for even higher redshift absorbers. Such systems provide the opportunity to measure isotopic ratios with high precision at a range of redshifts, which allows us to probe chemical enrichment timescales, and hence the sources of various isotopes, and provide observational constraints on models of galactic chemical evolution. 


\section{Bibliography}

Aitken, D. K., Smith, C. H., James, S. D., et al. 1988, MNRAS, 235, 19P

Barlow, M. J., Krause, O., Swinyard, B. M., et al. 2010, A\&A, 518, L138

Beelen, A., Cox, P., Benford, D. J., et al. 2006, ApJ, 642, 694

Bernes, C. 1979, A\&A, 73, 67

Bertoldi, F., Carilli, C. L., Cox, P., et al. 2003, A\&A, 406, L55

Biggs, A. D., Browne, I. W. A., Muxlow, T. W. B., \& Wilkinson, P. N. 2001, MNRAS, 322, 821

Borkowski, K. J. \& Shull, J. M. 1990, ApJ, 348, 169

Castro-Carrizo, A., Quintana-Lacaci, G., Bujarrabal, V., Neri, R., \& Alcolea, J. 2007, A\&A, 465, 457

Cherchneff, I. \& Dwek, E. 2009, ApJ, 703, 642

Combes, F. \& Wiklind, T. 1996, in Astrophysics and Space Science Library, Vol. 206, Cold Gas at High Redshift, ed. M. N. Bremer \& N. Malcolm, 215

Danziger, I. J., Fosbury, R. A. E., Alloin, D., et al. 1987, A\&A, 177, L13

de Jager, C. 1998, A\&A Rev., 8, 145

de Jager, C. \& Nieuwenhuijzen, H. 1997, MNRAS, 290, L50

Dinh-V.-Trung, Muller, S., Lim, J., Kwok, S., \& Muthu, C. 2009, ApJ, 697, 409

Gall, C., Hjorth, J., \& Andersen, A. C. 2011, A\&A Rev., 19, 43

Garcia-Segura, G., Langer, N., \& Mac Low, M.-M. 1996, A\&A, 316, 133

Hartmann, D. H., Predehl, P., Greiner, J., et al. 1997, Nuclear Physics A, 621, 83 
Henize, K. G. 1976, ApJS, 30, 491

Henkel, C., Jethava, N., Kraus, A., et al. 2005, A\&A, 440, 893

Hutsemékers, D., Cox, N. L. J., \& Vamvatira-Nakou, C. 2013, A\&A, 552, L6

Kamenetzky, J., McCray, R., Indebetouw, R., et al. 2013, ApJ, 773, L34

Kobayashi, C., Karakas, A. I., \& Umeda, H. 2011, MNRAS, 414, 3231

Kotak, R., Meikle, P., Pozzo, M., et al. 2006, ApJ, 651, L117

Kotak, R., Meikle, P., van Dyk, S. D., Höflich, P. A., \& Mattila, S. 2005, ApJ, 628, L123

Kotak, R., Meikle, W. P. S., Farrah, D., et al. 2009, ApJ, 704, 306

Kroupa, P. 2008, in Astronomical Society of the Pacific Conference Series, Vol. 390, Pathways Through an Eclectic Universe, ed. J. H. Knapen, T. J. Mahoney, \& A. Vazdekis, 3

Lagadec, E., Zijlstra, A. A., Oudmaijer, R. D., et al. 2011, A\&A, 534, L10

Le Bertre, T., Heydari-Malayeri, M., Epchtein, N., Gouiffes, C., \& Perrier, C. 1989, A\&A, 225, 417

Lucy, L. B., Danziger, I. J., Gouiffes, C., \& Bouchet, P. 1989, in Lecture Notes in Physics, Berlin Springer Verlag, Vol. 350, IAU Colloq. 120: Structure and Dynamics of the Interstellar Medium, ed. G. Tenorio-Tagle, M. Moles, \& J. Melnick, 164

Matsuura, M., Dwek, E., Meixner, M., et al. 2011, Science, 333, 1258

Menten, K. M., Carilli, C. L., \& Reid, M. J. 1999, in Astronomical Society of the Pacific Conference Series, Vol. 156, Highly Redshifted Radio Lines, ed. C. L. Carilli, S. J. E. Radford, K. M. Menten, \& G. I. Langston, 218

Milisavljevic, D. \& Fesen, R. A. 2013, ApJ, 772, 134

Moseley, S. H., Dwek, E., Glaccum, W., Graham, J. R., \& Loewenstein, R. F. 1989, Nature, 340, 697

Müller, H. S. P., Muller, S., Schilke, P., et al. 2015, A\&A, 582, L4

Muller, S., Beelen, A., Guélin, M., et al. 2011, A\&A, 535, A103

Muller, S., Black, J. H., Guélin, M., et al. 2014, A\&A, 566, L6

Muller, S., Guélin, M., Combes, F., \& Wiklind, T. 2007, A\&A, 468, L53 
Muller, S., Guélin, M., Dumke, M., Lucas, R., \& Combes, F. 2006, A\&A, 458, 417

Oudmaijer, R. D., Davies, B., de Wit, W.-J., \& Patel, M. 2009, in Astronomical Society of the Pacific Conference Series, Vol. 412, The Biggest, Baddest, Coolest Stars, ed. D. G. Luttermoser, B. J. Smith, \& R. E. Stencel, 17

Patnaik, A. R., Browne, I. W. A., King, L. J., et al. 1993, MNRAS, 261, 435

Poglitsch, A., Waelkens, C., Geis, N., et al. 2010, A\&A, 518, L2

Rho, J., Jarrett, T. H., Reach, W. T., Gomez, H., \& Andersen, M. 2009, ApJ, 693, L39

Rho, J., Kozasa, T., Reach, W. T., et al. 2008, ApJ, 673, 271

Rho, J., Onaka, T., Cami, J., \& Reach, W. T. 2012, ApJ, 747, L6

Roueff, E., Loison, J. C., \& Hickson, K. M. 2015, A\&A, 576, A99

Salpeter, E. E. 1955, ApJ, 121, 161

Sarangi, A. \& Cherchneff, I. 2013, ApJ, 776, 107

Sarangi, A. \& Cherchneff, I. 2015, A\&A, 575, A95

Schöier, F. L. \& Olofsson, H. 2001, A\&A, 368, 969

Sugerman, B. E. K., Ercolano, B., Barlow, M. J., et al. 2006, Science, 313, 196

Szalai, T. \& Vinkó, J. 2013, A\&A, 549, A79

Tielens, A. G. G. M. 2005, The Physics and Chemistry of the Interstellar Medium

Todini, P. \& Ferrara, A. 2001, MNRAS, 325, 726

van der Tak, F. F. S., Black, J. H., Schöier, F. L., Jansen, D. J., \& van Dishoeck, E. F. 2007, A\&A, 468, 627

Wiklind, T. \& Combes, F. 1995, A\&A, 299, 382

Wiklind, T. \& Combes, F. 1996, Nature, 379, 139

Wilson, T. L. 2009, Introduction to Millimeter/Sub-Millimeter Astronomy. ArXiv e-print, to be published in the Saas Fee Winter School 2008.

Yang, B., Stancil, P. C., Balakrishnan, N., \& Forrey, R. C. 2010, ApJ, 718, 1062 
